This item was submitted to Loughborough's Research Repository by the author.

Items in Figshare are protected by copyright, with all rights reserved, unless otherwise indicated.

\title{
Optimization of a main landing gear locking mechanism using bifurcation analysis
}

\section{PLEASE CITE THE PUBLISHED VERSION}

https://doi.org/10.2514/1.C034228

\section{PUBLISHER}

(c) American Institute of Aeronautics and Astronautics

\section{VERSION}

AM (Accepted Manuscript)

\section{PUBLISHER STATEMENT}

This work is made available according to the conditions of the Creative Commons Attribution-NonCommercialNoDerivatives 4.0 International (CC BY-NC-ND 4.0) licence. Full details of this licence are available at: https://creativecommons.org/licenses/by-nc-nd/4.0/

\section{LICENCE}

CC BY-NC-ND 4.0

\section{REPOSITORY RECORD}

Yin, Yin, Simon A. Neild, Jason Z. Jiang, James A.C. Knowles, and Hong Nie. 2017. "Optimization of a Main Landing Gear Locking Mechanism Using Bifurcation Analysis”. figshare. https://hdl.handle.net/2134/26251. 


\title{
Optimization of a Main Landing Gear Locking Mechanism Using Bifurcation Analysis
}

\author{
Yin Yin ${ }^{1}$ \\ Nanjing University of Aeronautics and Astronautics, Nanjing, Jiangsu, 210016, China \\ University of Bristol, Bristol, BS8 1TR, United Kingdom \\ Simon A. Neild ${ }^{2}$ and Jason Zheng Jiang ${ }^{3}$ \\ University of Bristol, Bristol, BS8 1TR, United Kingdom \\ James A. C. Knowles ${ }^{4}$ \\ Loughborough University, Leicestershire, England LE11 3TU, United Kingdom \\ and \\ Hong $\mathrm{Nie}^{5}$ \\ Nanjing University of Aeronautics and Astronautics, Nanjing, Jiangsu, 210016, China
}

\begin{abstract}
A key part of the main landing gear (MLG) of a civil aircraft is its locking mechanism that holds the gear in the deployed or down-locked state. The locking is driven by a spring mechanism and its release by the unlock actuator. This paper considers this mechanism in terms of its stability and the locking and unlocking forces required for down-locking. To study this an analytical model was developed. The equations, consisting of geometric constraints and force/moment equilibriums, were derived using the coordinate transformation method. Using numerical continuation to solve these equations, the effect of the unlock force on the MLG retraction cycle was analyzed. The variation of a fold bifurcation point, which indicates the transition between the locked state and the unlocked state, gives further insight into the required unlock force that governs the sizing of the unlock actuator. Moreover, some important information, such as the critical position for the lock-links' stops, the unlock position and the unlock force, are discussed using the bifurcation diagrams for the MLG retraction/extension cycle. Then, the effect of three key geometry parameters of the locking spring (the spring stiffness, unstrained spring length and spring attachment point) on the critical over-center angle and the unlock force are investigated. Finally, an optimization of the critical unlock force is carried out with a constraint on the initial over-center angle. The results show that the spring parameters have significant effects on the MLG's retraction performance. A 37\% reduction of the required unlock force is obtained through optimizing for the gear considered here.
\end{abstract}

\footnotetext{
${ }^{1}$ Ph.D. Student, College of Aerospace Engineering/Department of Mechanical Engineering, yinyinjordan@163.com.

${ }^{2}$ Professor of Dynamic and Control, Department of Mechanical Engineering, simon.neild@ bristol.ac.uk.

${ }^{3}$ Lecturer of Dynamic and Control, Department of Mechanical Engineering, z.jiang @ bristol.ac.uk.

${ }^{4}$ Lecturer of Dynamic and Control, Department of Aeronautical and Automotive Engineering.

${ }^{5}$ Professor of of Dynamic and Control, College of Aerospace Engineering, hnie@ nuaa.edu.cn.
} 


\section{Nomenclature}

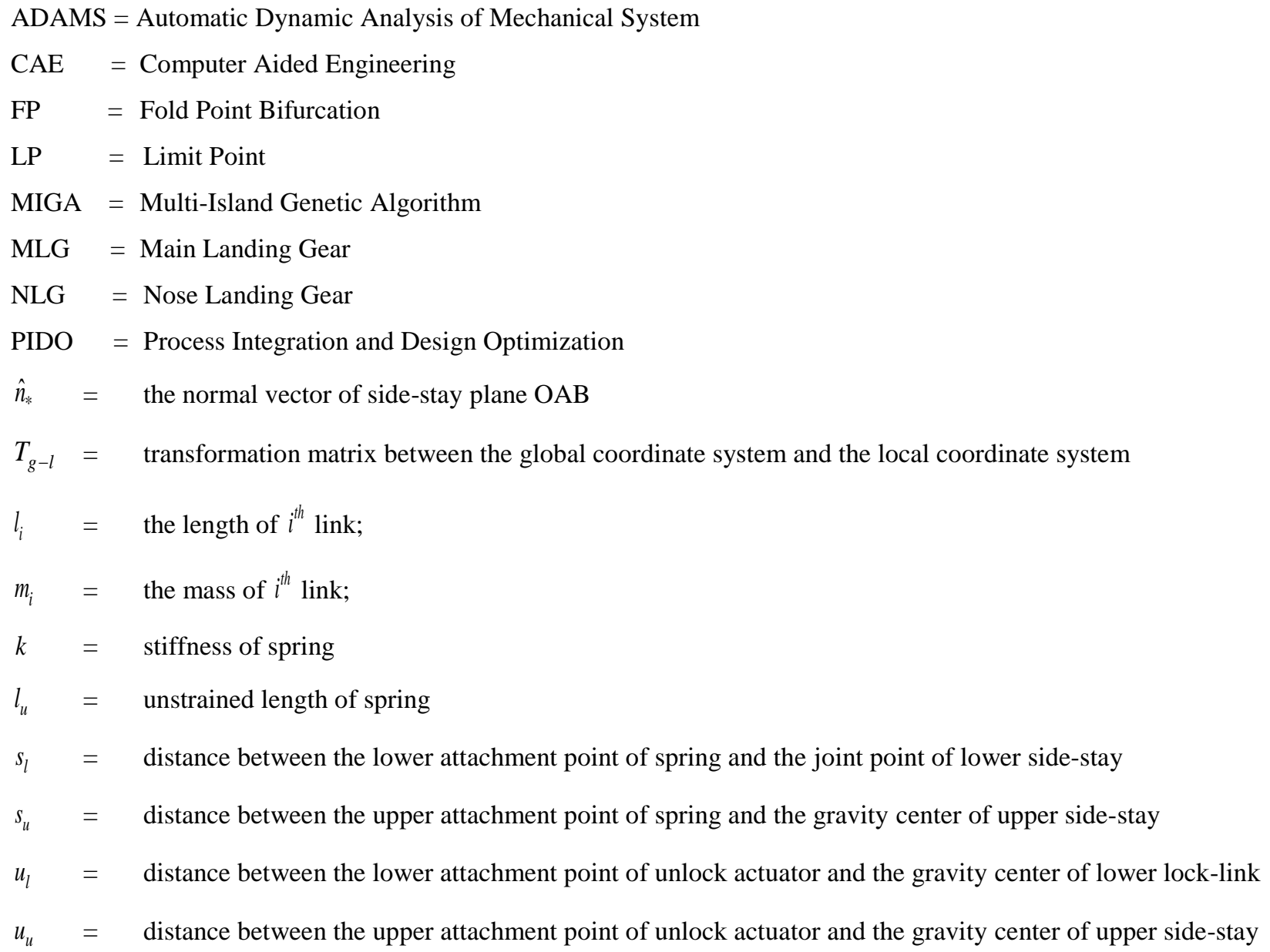

\section{Introduction}

$\mathrm{A}_{\mathrm{N}}$ aircraft main landing gear (MLG) is a complex mechanism that must be capable of being locked in both the deployed and retracted states. Typically, in the down-lock state the main strut is supported with a two-element sidestay that is locked in place using lock-links [1]. The lock-links "snap" into the locked state by passing the overcenter point and hitting stops which prevent further movement [2]. A spring is used to trigger the snapping and the mechanism can only be released from its down-locked state, allowing retraction of the landing gear, using an unlock actuator. Once unlocked, the landing gear can be retracted and fixed in the retracted state using an up-lock mechanism [1,3]. Dynamic simulation can be an effective method for the performance analysis of landing gear retraction mechanisms in the aviation industry $[4,5]$. Commercial dynamic simulation software (such as ADAMS, Siemens Virtual Lab and SIMPACK) [3, 6, 7] is typically used to establish a time domain dynamic simulation model of the landing gear's locking mechanism, which allows investigation of the changes of steady-state solutions, namely bifurcation points, via applying slowly-varying forces on the lock links. However, the time-domain dynamic 
simulation approach is inefficient for finding the steady-state solutions because a new simulation must be carried out for each parameter value being changed. For these investigations, dynamic simulations require a large amount of computation time.

An alternative approach is to make use of dynamic system theory [8] to study the bifurcations and topological changes in the solution space as parameters vary, by using a numerical continuation solver [9-11]. The key to the efficiency of numerical continuation is that it can be used to follow key features, such as bifurcation points, directly in the parameter space of interest with standard numerical continuation software such as COCO [12]. Bifurcation analysis and numerical continuation method have been widely applied to aircraft dynamics analysis for many years, as discussed in the review by Sharma et al. [13]. For example, landing gear shimmy oscillations have been studied to identify regions in which the only stable steady-state solution is that where these is no shimmy motion and their boundaries with regions where shimmy exists considering two key parameters: the forward velocity of aircraft and the vertical force acting on the landing gear [14-16]. Similarly, the characterization of aircraft ground handling behaviors has been studied using bifurcation analysis method. Bifurcation diagrams in terms of the aircraft velocity and the steering angle were obtained by conducting two-parameter continuations of saddle-node and Hopf bifurcations [17-19].

Regarding the continuation analysis of landing gear mechanism, it is necessary to assume that the retraction/deployment motion is sufficiently slow to be considered as quasi-static. This assumption was shown to be acceptable when compared to full dynamic models solved using time-stepping method - both the dynamic simulation and static equations exhibit limit points (LPs) in the locking mechanism: the prior method provides a dynamic result of jump at the limit point, while the later one gives a Fold Point (FP) bifurcation point [2]. Using this approach, an analysis of the "snapping" of the MLG lock-links into the deployed state has been conducted using bifurcation theory and the effects of geometry parameters on the locking performance studied by tracing key fold points in parameter space $[2,20]$. This method will be used for present study.

The snapping of the lock-links into the locked position is observed as a jump, triggered by a fold in the solution surface, from the stable solution branch of the above-over-center state to the stable branch of the below-over-center state. Unlocking the mechanism from the locked below-over-center state requires use of the unlock actuator, which drives the lock-links back into the unlocked above-over-center state. The retraction actuator is then able to move the landing gear between the deployed and retracted states. The unlock force has a significant effect on the retraction 
trajectory of the landing gear, and is affected by multiple parameters such as the unstrained length of lock spring, the spring stiffness and the spring attachment point. In this work, the unlock force is studied by (1) analyzing the effects of different unlock forces on the retraction performance of the landing gear and (2) analyzing the effects of different geometric parameters on the unlock force.

To this end, the structure of this paper is organized as follows. A set of coupled kinematic and force equilibrium equations for a typical three-dimensional (3-D) retraction MLG is derived in Section 2. The retraction and deployment cycle, using the nominal MLG parameter values, is described in Section 3. In Section 4, the effects of applying different unlock forces on the MLG retraction trajectory are analyzed in detail and two important features, the critical unlock position and the critical unlock force, are defined. Following this, in Section 5, the effects of selecting different geometric parameters on the required unlock force are analyzed, allowing parameters to be optimized for a lower unlock force and hence a reduced specification for the unlock actuator. Finally, Section 6 provides some conclusions.

\section{Mechanism and Equations}

A spatial-retraction MLG shown in Fig. 1 consists of five links. The main strut is a buffer mechanism for absorbing vertical energy during the landing process and supporting the aircraft during ground maneuvers. The upper and lower side-stays are the main parts of supporting structures. The MLG has two separate locking mechanisms to fix the main strut in the retracted and deployed states: one is the down-lock mechanism, consisting of upper and lower lock-links; the other is a hook lock box which is applied as the up-lock mechanism (not shown). Three actuators are needed to realize the movements of retraction and deployment: two unlock actuators are required to unlock the two locking mechanisms and the retraction actuator is used to move the MLG between the deployed and retracted states. This work focuses on the down-locking mechanism, namely the lock-links, rather than the hook lock box device, as the latter device has little effect on the MLG retraction performance.

\section{A. Definition of Coordinate System}

In previous studies a two-dimensional (2D) retraction landing gear, which can be analyzed as a planar linkage mechanism problem without transformation between different coordinate systems, has been considered [2]. Compared to this the 3D retraction MLG is much more complicated because the side-stays and lock-links follow a compound motion consisting of fold and rotation. In addition, the two moving planes of side-stays and main strut are 
non-coplanar, which leads to difficulties in the kinematic and dynamic analysis of retraction mechanisms. However, an interesting motion law, which states side-stays and lock-links are always in the same plane during the whole retraction or deployment process, makes it possible to reduce the complexity $[4,5]$. Considering the side-stays and main strut in their own local coordinate systems, the spatial problem is changed to two planar problems with the variables being expressed in the different coordinates and linked via a transformation matrix.

Based on the above analysis, the global coordinate system $O-X Y Z$ is set up as shown in Fig.1. The origin $O$ is at the cross point of the three rotation axes. The $O X$ axis is defined as the rotation shaft of the main strut and points in the direction of aircraft forward direction, the $O Z$ axis points vertically downwards and the $O Y$ axis is decided by the right-hand rule. The local coordinate system for the side-stays, $O-x y z$, is defined as follows: the $O y$ axis is collinear with the rotation axis of the upper node; the $O z$ axis is perpendicular to the $O y$ axis in the side-stay plane and points downward; and the $O x$ axis completes the right-handed coordinate system.
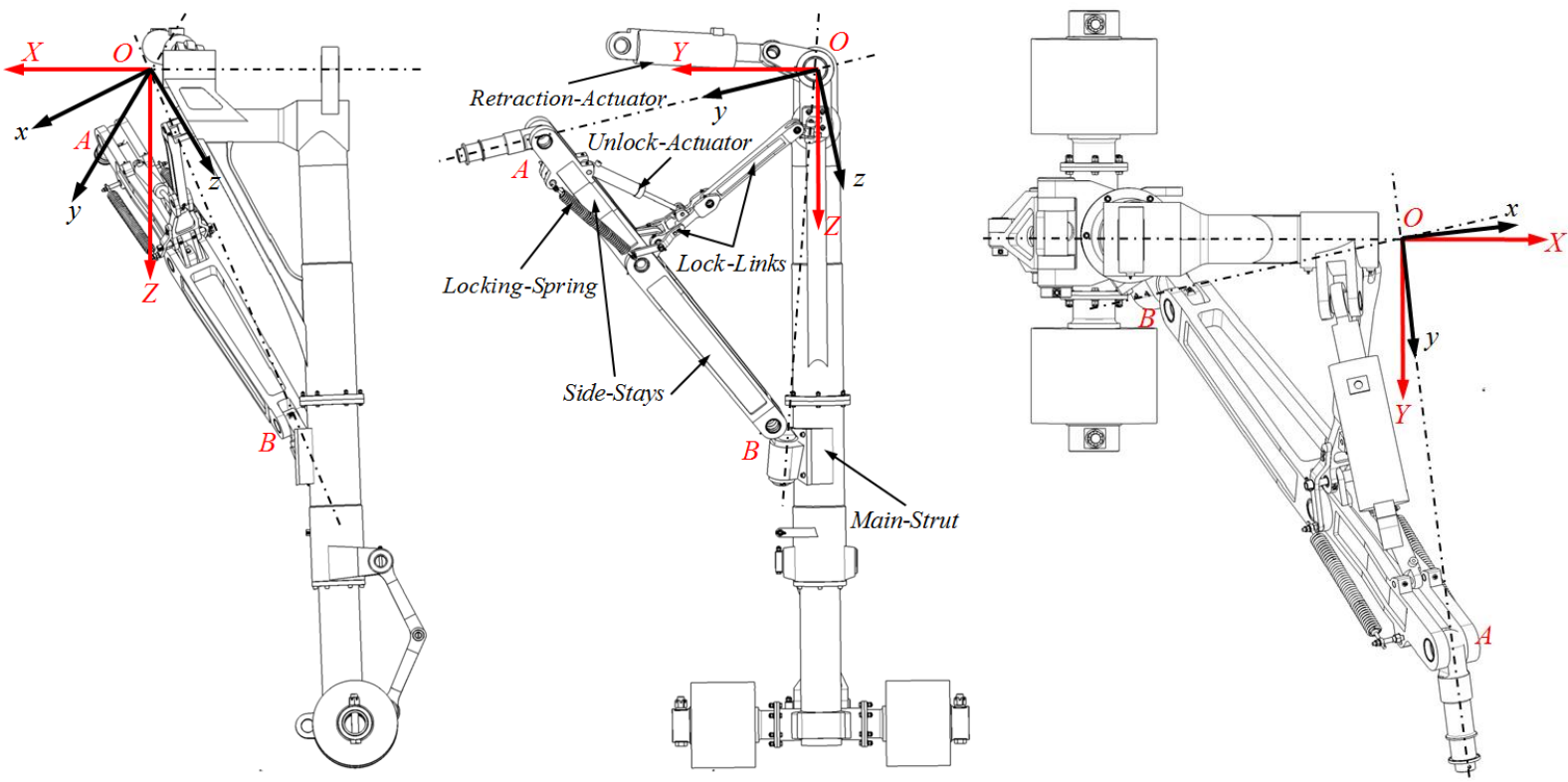

Fig. 1 Definition of coordinate system

\section{B. Coordinate Transformation}

The transformation matrix $T_{g-l}^{\prime}$ between the global coordinate system and the local coordinate system for the down-lock position (as shown in Fig. 2) can be obtained using the Carl Dan coordinate transformation method [8]. The order of the Carl Dan rotations is as follows. The global coordinate $[X, Y, \mathrm{Z}]$ system begins with a rotation of $\alpha_{g-l}$ about the OX-axis, then a rotation $\beta_{g-l}$ about the new OY'-axis, and finally a rotation of $\gamma_{g-l}$ about the 
resulting OZ"-axis into the local coordinate system $[x, y, z]$. The resulting transform is given by

$$
T_{g-l}^{\prime}=\left[\begin{array}{ccc}
c \beta_{g-l} \cdot c \gamma_{g-l} & -c \beta_{g-l} \cdot s \gamma_{g-l} & s \beta_{g-l} \\
s \alpha_{g-l} \cdot s \beta_{g-l} \cdot c \gamma_{g-l}+c \alpha_{g-l} \cdot s \gamma_{g-l} & -s \alpha_{g-l} \cdot s \beta_{g-l} \cdot s \gamma_{g-l}+c \alpha_{g-l} \cdot c \gamma_{g-l} & -s \alpha_{g-l} \cdot c \beta_{g-l} \\
-c \alpha_{g-l} \cdot s \beta_{g-l} \cdot c \gamma_{g-l}+s \alpha_{g-l} \cdot s \gamma_{g-l} & c \alpha_{g-l} \cdot s \beta_{g-l} \cdot s \gamma_{g-l}+s \alpha_{g-l} \cdot c \gamma_{g-l} & c \alpha_{g-l} \cdot c \beta_{g-l}
\end{array}\right],
$$

where the shorthand $s \equiv \sin$ and $c \equiv \cos$ has been used.

The local coordinate system will be rotating about $O y$ axis as the MLG retracts, that is to say, the down-lock position transformation matrix, $T_{g-l}^{\prime}$, is followed by transformation matrix $T_{\theta_{1}}$, which is given by

$$
T_{\theta_{1}}=\left[\begin{array}{ccc}
\cos \left(\eta_{g-l}\right) & 0 & \sin \left(\eta_{g-l}\right) \\
0 & 1 & 0 \\
-\sin \left(\eta_{g-l}\right) & 0 & \cos \left(\eta_{g-l}\right)
\end{array}\right]
$$

Where $\eta_{g-l}$ indicates the rotation angle of the local coordinate system about the $O y$ axis.

The rotation angle $\eta_{g-l}$, can be obtained via calculating the angle change of the normal vector of plane OAB using

$$
\eta_{g-l}=\cos ^{-1}\left(\frac{\hat{n}_{0} \cdot \hat{n}_{\theta_{1}}}{\left|\hat{n}_{0}\right| \cdot\left|\hat{n}_{\theta_{1}}\right|}\right),
$$

where $\hat{n}_{0}$ indicates the initial normal vector of plane $\mathrm{OAB} ; \hat{n}_{\theta_{1}}$ denotes the normal vector of plane $\mathrm{OAB}$ with a rotation angle $\theta_{1}$ of the main strut. The final transformation matrix $T_{g-l}$ can now be written as:

$$
T_{g-l}=T_{\theta_{1}} \cdot T_{g-l}^{\prime} .
$$

\section{Model Formulation}

Before the geometric constraint and equilibrium equations are discussed, it is worth considering how the model will be constructed in terms of the unknowns and the equations relating them. The model will be derived without the inclusion of the lock-link stops, the effect of which can be imposed on the mechanism later as a constraint.

There are five elements in the mechanism, the locations of which can be described by $\left\{x_{i}, y_{i}, z_{i}, \theta_{i}\right\}$ in the local coordinate axis, where $\theta_{i}$ is the angle of the element in the y-axis. Alongside these 20 degrees-of-freedom there are 19 geometric constraint equations. This indicates that the mechanism layout can be described completely by one angle or location. In addition, there are 39 forces and force balance equations. These forces are made up of 30 interlink forces ( $x, y$ and $z$ directions for either side of the 5 inter-link joints), 6 ground-link forces from the 2 external supports, a spring force and an unlock actuator force, as well as a moment applied by the retraction actuator. Alongside these there are 39 force and moment balance equations. 
The result is that, for a given retraction actuator moment and unlock actuator force, all the other forces (or a subset of them) and the mechanism position can be found.

\section{Geometric Constraints}

According to the principle of $3 \mathrm{D}$ retraction mechanism, joints $\mathrm{O}, \mathrm{C}, \mathrm{D}$, and $\mathrm{E}$ are revolute joints, whilst joints $\mathrm{A}$, $\mathrm{B}$ and $\mathrm{H}$ are universal joints. This indicates that the degree of freedom of the retraction mechanism equals 1 . Both the side-stays and the main strut can be considered as planar problems based on the definition of their coordinate systems, thus each link can be described in terms of four degrees-of-freedom, $L_{i}=\left\{x_{i}, y_{i}, z_{i}, \theta_{i}\right\}$ or $L_{i}=\left\{X_{i}, Y_{i}, Z_{i}, \theta_{i}\right\}$, as shown in Fig. 2.

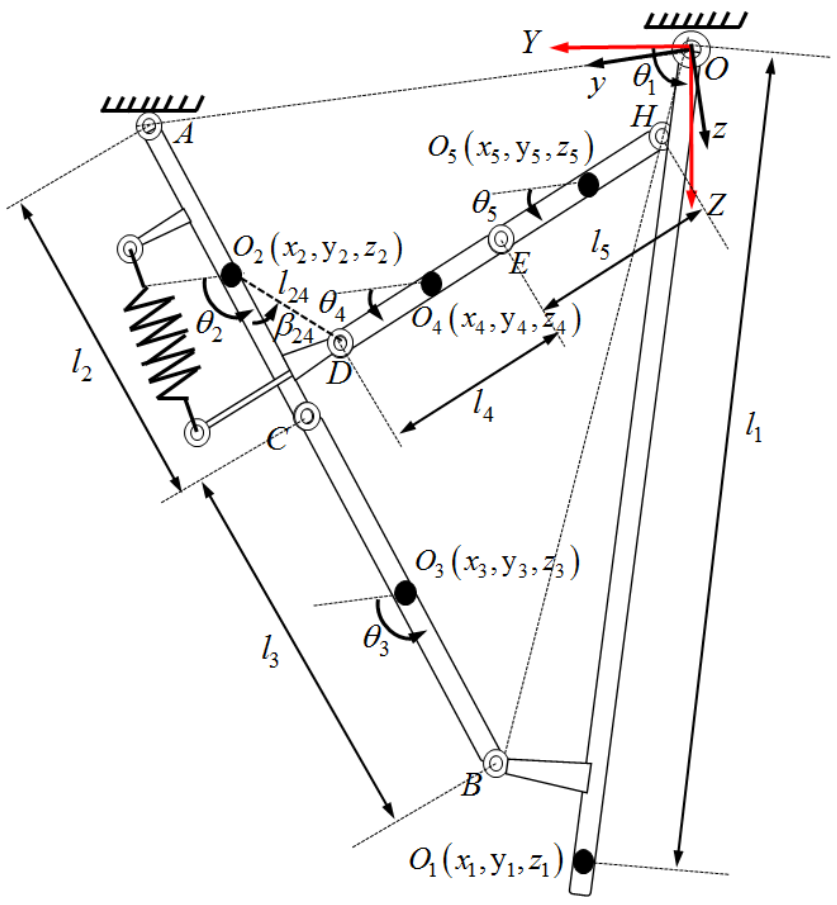

Fig. 2 Sketch of spatial retraction mechanism

$\left(x_{i}, y_{i}, z_{i}\right)$ and $\left(X_{i}, Y_{i}, Z_{i}\right)$ are the local coordinates and the global coordinates respectively, both of which describe the position of $L_{i}$ 's center of gravity. The local and global coordinate systems are related via the transformation matrix $T_{g-l} . \theta_{i}$ indicates the relative angle between the $i^{\text {th }}$ link and y axis.

Given the conditions of 5 links, 20 states and 1degree of freedom, 19 geometric constraint equations are needed to describe the physical constrains in the landing gear mechanism. The geometric constraints can be expressed as follows: 


$$
\left[\begin{array}{c}
X_{1}+C \\
Y_{1}-l_{1} \cdot \sin \left(\theta_{1}\right) \\
Z_{1}-l_{1} \cdot \cos \left(\theta_{1}\right) \\
x_{2} \\
y_{2}-\frac{l_{2}}{2} \cdot \cos \left(\theta_{2}\right)-A_{y} \\
z_{2}-\frac{l_{2}}{2} \cdot \sin \left(\theta_{2}\right)-A_{z} \\
x_{3} \\
y_{2}+\frac{l_{2}}{2} \cdot \cos \left(\theta_{2}\right)-\left(y_{3}-\frac{l_{3}}{2} \cdot \cos \left(\theta_{3}\right)\right) \\
z_{2}+\frac{l_{2}}{2} \cdot \sin \left(\theta_{2}\right)-\left(z_{3}-\frac{l_{3}}{2} \cdot \sin \left(\theta_{3}\right)\right) \\
z_{3}-\frac{l_{5}}{2} \cdot \sin \left(\theta_{5}\right)-H_{z} \\
y_{3}+\frac{l_{3}}{2} \cdot \cos \left(\theta_{3}\right)-B_{y} \\
z_{4}+\frac{l_{3}}{2} \cdot \sin \left(\theta_{3}\right)-B_{z} \\
z_{4}+\frac{l_{5}}{2} \cdot \sin \left(\theta_{5}\right)-\left(z_{4}-\frac{l_{4}}{2} \cdot \sin \left(\theta_{4}\right)\right) \\
x_{4}+\frac{l_{4}}{2} \cdot \cos \left(\theta_{4}\right)-\left(\theta_{2}+\frac{l_{24}}{2} \cdot \sin \left(\beta_{24}+\theta_{2}\right)\right.
\end{array}\right]
$$

Here $B_{y}$ and $B_{z}$ are the $y$ value and $z$ value (respectively) of point B under the local coordinate system. They can be calculated by the expression: $\left[\begin{array}{c}B_{x} \\ B_{y} \\ B_{z}\end{array}\right]=T_{g-l} \cdot\left[\begin{array}{c}B_{X} \\ B_{Y} \\ B_{Z}\end{array}\right]$. Similarly, $H_{y}, H_{z}$ and $A_{y}, A_{z}$ are the y value and z value of points $\mathrm{H}$ and $\mathrm{A}$ under the local coordinate system respectively.. $[C]$ indicates the $\mathrm{X}$ value of the center of the main strut's gravity in the global coordinate system.

\section{E. Force/ Moment Equilibrium Equations}

The force elements of the MLG consists of: 
- $F_{i ; j}^{x}, F_{i ; j}^{y}, F_{i ; j}^{z}$ indicate the internal force between adjacent two links, with $\mathrm{i}$ and $\mathrm{j}$ denoting the link that is experiencing the force and the link that is applying the force respectively. 30 parameters are needed to describe the internal forces;

- 6 ground-link forces: these are the internal forces between the strut and the aircraft body, and the internal forces between the upper side-stay and the aircraft body;

- $F_{s}$ indicates the lock spring force;

- $F_{u}$ denotes the unlock actuator force;

- $M_{a c t}$ is the retraction actuator moment.

Considering all 39 forces of the retraction mechanism increases the computational complexity, so some simplifications are put forward here. Firstly, half the internal joint reaction forces can be removed by describing them as equal and opposite ( $\operatorname{such}$ as $F_{3 ; 1}^{x}=-F_{1 ; 3}^{x}$ ). As the side-stays, lock-links and main strut are planar (as shown in Fig. 3(a) and 3(b)), in general the x components of the internal forces are not needed. There are two exceptions to this due to the fact that the force elements $\left[F_{1 ; 5}^{Y}, F_{1 ; 5}^{Z}\right]$ and $\left[F_{1 ; 3}^{Y}, F_{1 ; 3}^{Z}\right]$ are needed to calculate the moment equilibrium of the main strut (as shown in Fig. 3(b)). This requires the full local coordinate force elements, hence $F_{3 ; 1}^{x}$ and $F_{5 ; 1}^{x}$ are needed. Knowles et al. [2] points out that most of the ground-link forces can be removed as they are of no interest. The exception here is the x component of $F_{2 ; R A}, F_{2 ; R A}^{x}$, which is needed to establish the mechanism equilibrium equations in the $\mathrm{x}$ direction combined with the force elements $F_{3 ; 1}^{x}$ and $F_{5 ; 1}^{x}$ (as shown in Fig. 3(c)). In addition, the lock spring force, which can be determined by the geometric constraints and Hooke's Law, can be easily eliminated. After eliminating these, 14 force state variables are left along with the retraction actuator moment. Treating the retraction actuator moment and unlock actuator force as inputs, 14 force and moment balance equations can be derived (detailed below) allowing the forces and the mechanism position at equilibrium to be found. 


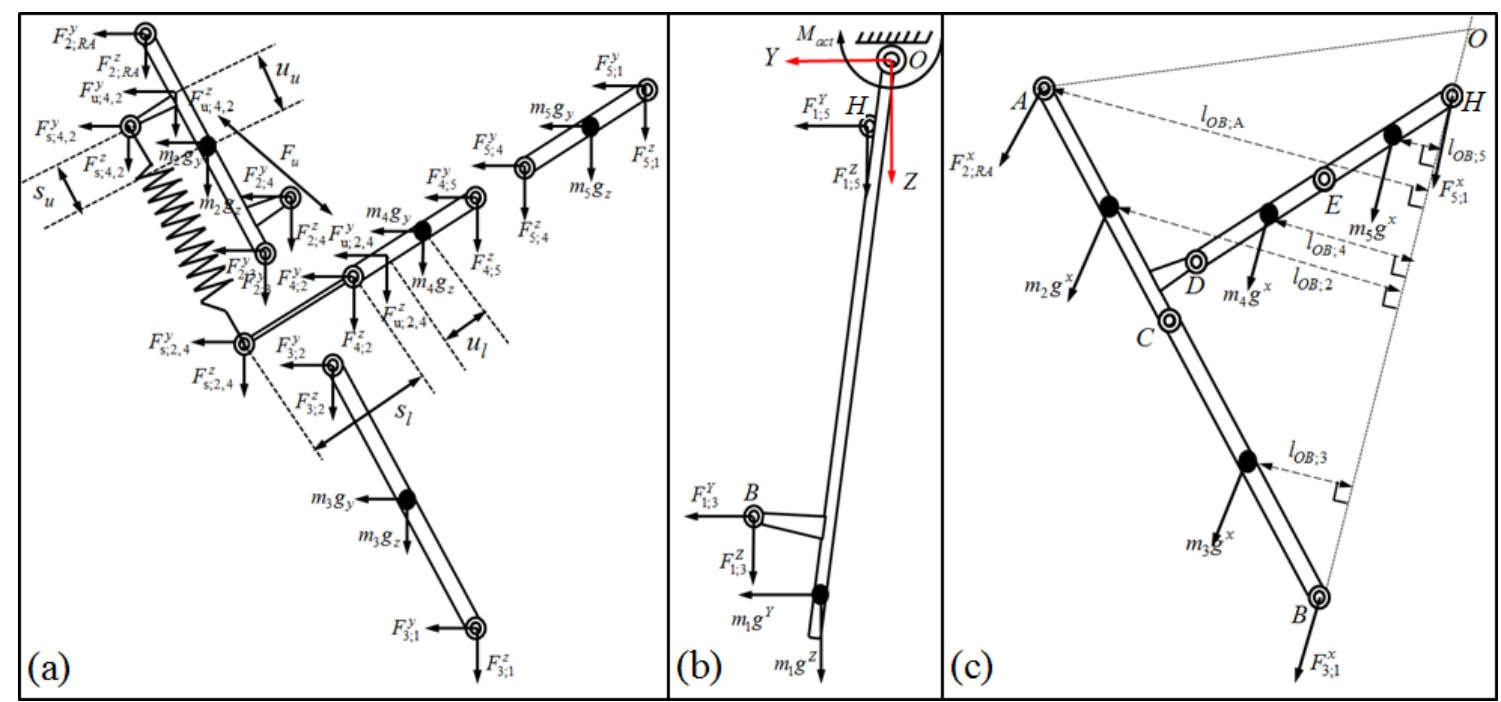

Fig. 3 Force diagram of spatial retraction mechanism.

(1) Force and moment equilibrium of the side-stays and lower lock link - 10 equations

Each of the links should be in force and moment equilibrium. The equilibrium equations can be written as:

$$
\left\{\begin{array}{l}
\sum_{j} F_{i ; j}^{y}=0 \\
\sum_{j} F_{i ; j}^{z}=0 \\
\sum_{j} M_{i ; j}^{*}=l_{i ; j}^{*} \cdot F_{i ; j}=0
\end{array},\right.
$$

where length $l_{i, j}^{*}$ is the moment arm of force $F_{i ; j}$ from position *.

Lock spring force and unlock force can be expressed as

$$
\text { Spring force: }\left\{\begin{array}{l}
F_{s}=\left(\sqrt{\left(y_{s}^{u p}-y_{s}^{l o w}\right)^{2}+\left(z_{s}^{u p}-z_{s}^{l o w}\right)^{2}}-l u\right) \cdot k \\
F_{s ; 2,4}^{y}=\frac{y_{s}^{l o w}-y_{s}^{u p}}{\sqrt{\left(y_{s}^{u p}-y_{s}^{l o w}\right)^{2}+\left(z_{s}^{u p}-z_{s}^{l o w}\right)^{2}}} \cdot F_{s} \\
F_{s ; 2,4}^{z}=\frac{z_{s}^{l o w}-z_{s}^{u p}}{\sqrt{\left(y_{s}^{u p}-y_{s}^{l o w}\right)^{2}+\left(z_{s}^{u p}-z_{s}^{l o w}\right)^{2}}} \cdot F_{s} \\
F_{s ; 2,4}^{y}=-F_{s ; 4,2}^{y} \\
F_{s ; 2,4}^{z}=-F_{s ; 4,2}^{z}
\end{array},\right.
$$

and 


$$
\text { Unlock force: }\left\{\begin{array}{l}
F_{u ; 2,4}^{y}=\frac{y_{u}^{\text {low }}-y_{u}^{u p}}{\sqrt{\left(y_{u}^{u p}-y_{u}^{\text {low }}\right)^{2}+\left(z_{u}^{u p}-z_{u}^{\text {low }}\right)^{2}}} \cdot F_{u} \\
F_{u ; 2,4}^{z}=\frac{z_{u}^{\text {low }}-z_{u}^{u p}}{\sqrt{\left(y_{u}^{u p}-y_{u}^{\text {low }}\right)^{2}+\left(z_{u}^{u p}-z_{u}^{\text {low }}\right)^{2}}} \cdot F_{u} . \\
F_{u ; 2,4}^{y}=-F_{u ; 4,2}^{y} \\
F_{u ; 2,4}^{z}=-F_{u ; 4,2}^{z}
\end{array}\right.
$$

Here $\left\{\left(y_{s}^{u p}, z_{s}^{u p}\right),\left(y_{s}^{\text {low }}, z_{s}^{\text {low }}\right)\right\}$ and $\left\{\left(y_{u}^{u p}, z_{u}^{u p}\right),\left(y_{u}^{\text {low }}, z_{u}^{\text {low }}\right)\right\}$ are the coordinates of the lock spring's upper and lower attachment points and the unlock actuator's upper and lower attachment points, respectively.

(2) Moment equilibrium of the main strut -1 equation

As shown in Fig. 3(b), the moment equilibrium equation of the main strut can be written as

$$
-F_{1 ; 3}^{Y} \cdot B_{Z}+F_{1 ; 3}^{Z} \cdot B_{Y}-F_{1 ; 5}^{Y} \cdot H_{Z}+F_{1 ; 5}^{Z} \cdot H_{Y}+m_{1} g \cdot Y_{1}-M_{a c t}=0 \text {. }
$$

Here $F_{1 ; 3}^{Y}, F_{1 ; 3}^{Z}$ represent the forces on the main strut applied by the lower side-stay, $F_{1 ; 5}^{Y}, F_{1 ; 5}^{Z}$ represent those on the main strut applied by the upper lock link and $M_{a c t}$ is the retraction actuator moment.

The inverse $T_{g-l}^{-1}$ of the transformation matrix $T_{g-l}$ is used to express the side-stay and lock-link local force $F_{1 ; 3}^{y}, F_{1 ; 3}^{z}$ and $F_{1 ; 5}^{y}, F_{1 ; 5}^{z}$ in the global coordinate system. The transformation expressions are given by

$$
\begin{aligned}
& {\left[\begin{array}{c}
F_{1 ; 3}^{X} \\
F_{1 ; 3}^{Y} \\
F_{1 ; 3}^{Z}
\end{array}\right]=T_{g-l}^{-1} \cdot\left[\begin{array}{l}
F_{1 ; 3}^{x} \\
F_{1 ; 3}^{y} \\
F_{1 ; 3}^{z}
\end{array}\right]=t \cdot\left[\begin{array}{c}
-F_{3 ; 1}^{x} \\
-F_{3 ; 1}^{y} \\
-F_{3 ; 1}^{z}
\end{array}\right],} \\
& {\left[\begin{array}{c}
F_{1 ; 5}^{X} \\
F_{1 ; 5}^{Y} \\
F_{1 ; 5}^{Z}
\end{array}\right]=T_{g-l}^{-1} \cdot\left[\begin{array}{l}
F_{1 ; 5}^{x} \\
F_{1 ; 5}^{y} \\
F_{1 ; 5}^{z}
\end{array}\right]=t \cdot\left[\begin{array}{l}
-F_{5 ; 1}^{x} \\
-F_{5 ; 1}^{y} \\
-F_{5 ; 1}^{z}
\end{array}\right] .}
\end{aligned}
$$

Here $T_{g-l}^{-1}=t$ denotes the inverse of the transformation matrix $T_{g-l}$. In order to get the specific expression of $F_{1 ; 3}^{Y}, F_{1 ; 3}^{Z}$ and $F_{1 ; 5}^{Y}, F_{1 ; 5}^{Z}$, equation (10) and (11) can be expanded as follows

$$
\left\{\begin{array}{l}
F_{1 ; 3}^{Y}=-\left(t_{2,1} \cdot F_{3 ; 1}^{x}+t_{2,2} \cdot F_{3 ; 1}^{y}+t_{2,3} \cdot F_{3 ; 1}^{z}\right) \\
F_{1 ; 3}^{Z}=-\left(t_{3,1} \cdot F_{3 ; 1}^{x}+t_{3,2} \cdot F_{3 ; 1}^{y}+t_{3,3} \cdot F_{3 ; 1}^{z}\right) \\
F_{1 ; 5}^{Y}=-\left(t_{2,1} \cdot F_{5 ; 1}^{x}+t_{2,2} \cdot F_{5 ; 1}^{y}+t_{2,3} \cdot F_{5 ; 1}^{z}\right) \\
F_{1 ; 5}^{Z}=-\left(t_{3,1} \cdot F_{5 ; 1}^{x}+t_{3,2} \cdot F_{5 ; 1}^{y}+t_{3,3} \cdot F_{5 ; 1}^{z}\right)
\end{array} .\right.
$$

(3) Force and moment equilibrium in the $\mathrm{x}$ direction -3 equations 
The force state variables contain only three unknown components in the $\mathrm{x}$ direction, namely, $F_{1 ; 3}^{x}, F_{1 ; 5}^{x}$ and $F_{2 ; \mathrm{RA}}^{x}$. As shown in Fig. 3(c), the three unknown state variables can be obtained by the equations as follows

$$
\left\{\begin{array}{l}
F_{2 ; R A}^{x} \cdot l_{O B ; A}+m_{2} g^{x} \cdot l_{O B ; 2}+m_{3} g^{x} \cdot l_{O B ; 3}+m_{4} g^{x} \cdot l_{O B ; 4}+m_{5} g^{x} \cdot l_{O B ; 5}=0 \\
F_{2 ; R A}^{x}+m_{2} g^{x}+m_{3} g^{x}+m_{4} g^{x}+m_{5} g^{x}+F_{3 ; 1}^{x}+F_{5 ; 1}^{x}=0 \\
F_{5 ; 1}^{x} \cdot\left(z_{5}-\frac{l_{5}}{2} \cdot \sin \theta_{5}\right)+F_{3 ; 1}^{x} \cdot\left(z_{3}+\frac{l_{3}}{2} \cdot \sin \theta_{3}\right)+m_{2} g^{x} \cdot z_{2}+m_{3} g^{x} \cdot z_{3}+m_{4} g^{x} \cdot z_{4}+m_{5} g^{x} \cdot z_{5}=0
\end{array} .\right.
$$

Here $l_{O B ; *}$ indicates the distance from point $*$ to axis $\overline{O B}$, namely the moment arm about axis $\overline{O B}$ and $z_{i}$ denotes the shortest length from $L_{i}$ 's center of gravity to axis $\overline{O A}$.

These force and moment equilibrium equations can now be assembled in the matrix form

$$
A \bar{F}-\bar{B}=0
$$

Where $\bar{F}$ is a vector of the inter link forces, $\mathrm{A}$ is a matrix of force coefficients, and $\bar{B}$ is a vector of the remaining terms (the spring, actuator and gravitational forces). These vectors and matrices are given in the Appendix.

\section{Retraction and Deployment Cycle}

In order to retract or deploy the MLG in an ordered manner, the retraction actuator must work with the unlock actuator. For the retraction process, an unlock actuator is needed to drive the lock-links to fold upwards and release first, then a retraction actuator is engaged to retract the MLG to the up-lock position. Usually, the unlock actuator can be switched off part-way through the retraction cycle. However, it is worth pointing out that the time at which this occurs has little effect on the retraction as the retraction actuator generates sufficient force to counteract the external loads [20]. Hence, in this work, the unlock actuator is switched off after the MLG has been locked in the retracted position, allowing the effects of the actuators' forces on the retraction performance to be studied separately. For the deployment process, the third actuator is engaged to unlock the up-lock mechanism (As this mechanism does not include the lock-links, it is not considered here). Once unlocked, the retraction actuator provides resistive moment to enable the main strut to deploy slowly. Finally, the MLG is locked at the down-lock position with the help of a spring force. Hence, a retraction schedule consists of an unlock stage, retraction stage, unlock force release stage and extension stage - this is shown in Fig. 4. Note that the timescales in Fig. 4 are arbitrary, the only real requirement is that the ramps are sufficiently slow for the inertial loading to be negligible. 


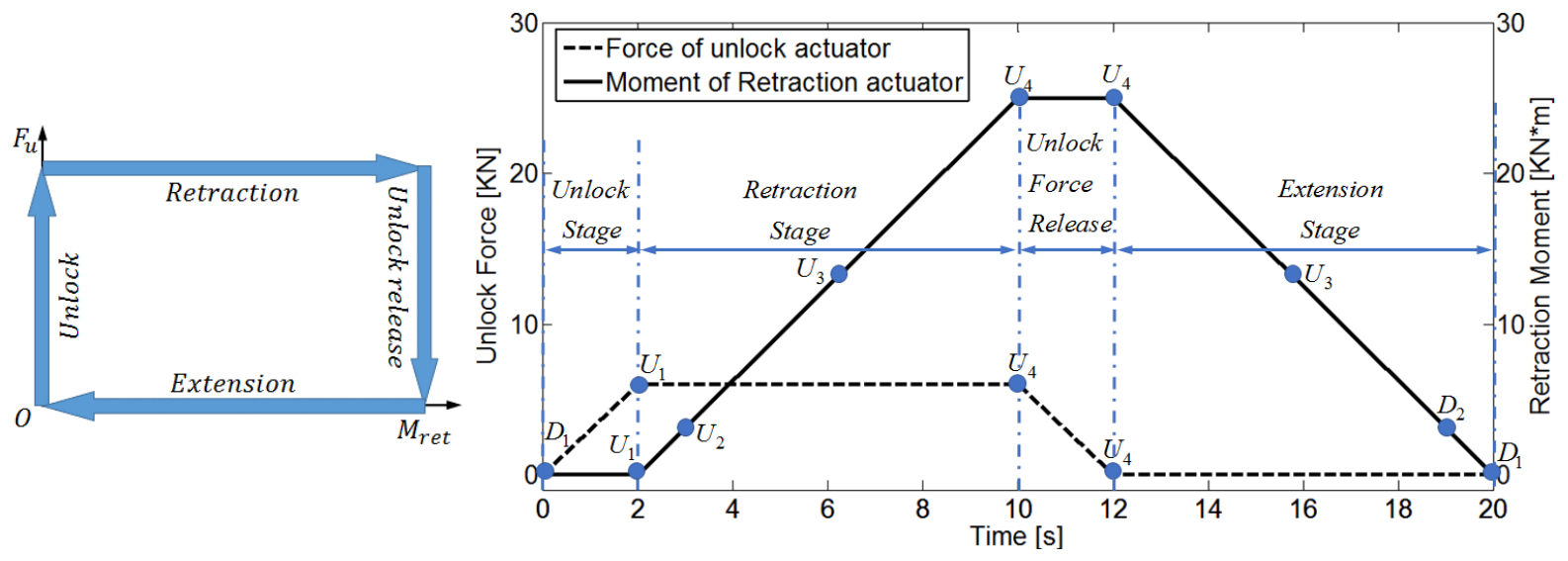

Fig.4 Schedule of landing gear retraction and extension

Given the key roles the unlock actuator force $F_{u}$ and the retraction moment $M_{a c t}$ play in the retraction and extension cycle, this cycle is now considered. The static equilibrium equations can be solved using the numerical continuation method, revealing how the over-center angle $\theta_{o v}$ varies as a function of the unlock actuator force $F_{u}$ and the retraction moment $M_{a c t}$. This over-center angle, $\theta_{o v}=\theta_{4}-\theta_{5}$, represents the angle between the two locklinks. Given the one degree-of-freedom nature of the mechanism, this angle uniquely describes the state of the gear, such that the retraction angle ${ }_{1}$, for example, can be calculated directly from it. Due to the geometry of the locklinks, which can rotate clockwise or anticlockwise relative to each other, the reverse cannot be calculated. The parameter values for the landing gear mechanism used in the simulations presented here are for a mid-size passenger aircraft and are given in Table 1.

Table 1 Main parameters of retraction mechanism

\begin{tabular}{cccccc}
\hline \hline Parameters & Values & Parameters & Values & Parameters & Values \\
\hline$l_{1}, m$ & 1.9224 & $m_{1}, k g$ & 1300.278 & $s_{u}, m$ & 0.2 \\
$l_{2}, m$ & 0.7575 & $m_{2}, k g$ & 73.197 & $u_{l}, m$ & 0.03 \\
$l_{3}, m$ & 1.1434 & $m_{3}, k g$ & 69.296 & $u_{u}, m$ & 0.1 \\
$l_{4}, m$ & 0.3184 & $m_{4}, k g$ & 10.492 & $\left(A_{X}, A_{Y}, A_{Z}\right), m$ & $(0.1531,1.2078,0.2933)$ \\
$l_{5}, m$ & 0.5115 & $m_{5}, k g$ & 7.788 & $\left(B_{X}, B_{Y}, B_{Z}\right)_{\theta_{1}=0}, m$ & $(-0.6717,0.1318,1.6257)$ \\
$\beta_{24}, d e g$ & 18.37 & $k, N / m$ & 6000 & $\left(F_{X}, F_{Y}, F_{Z}\right)_{\theta_{1}=0}, m$ & $(-0.1132,0.0222,0.2739)$ \\
$l_{24}, m$ & 0.3717 & $l_{u}, m$ & 0.34 & $\left(\alpha_{g-l}, \beta_{g-l}, \gamma_{g-l}\right), d e g$ & $(-10.35,25.01,7.75)$ \\
$C, m$ & 0.7971 & $s_{l}, m$ & 0.225 & -- & -- \\
\hline \hline
\end{tabular}


Fig. 5 shows the numerical continuation results of a typical retraction and extension cycle. The black curves represent the equilibrium solutions where the lock-links are above-over-center. The dark grey curves show the equilibrium solutions where the lock-links are below-over-center. The light grey curves denotes the MLG's response under the sole actuation of the unlock force. Furthermore, solid curves denote stable equilibrium solutions, while dashed curves indicate unstable equilibrium solutions. A fold bifurcation (labeled FP) occurs on the interface between the solid and dashed curves, and indicates the equilibrium solution changes stability via the FP.

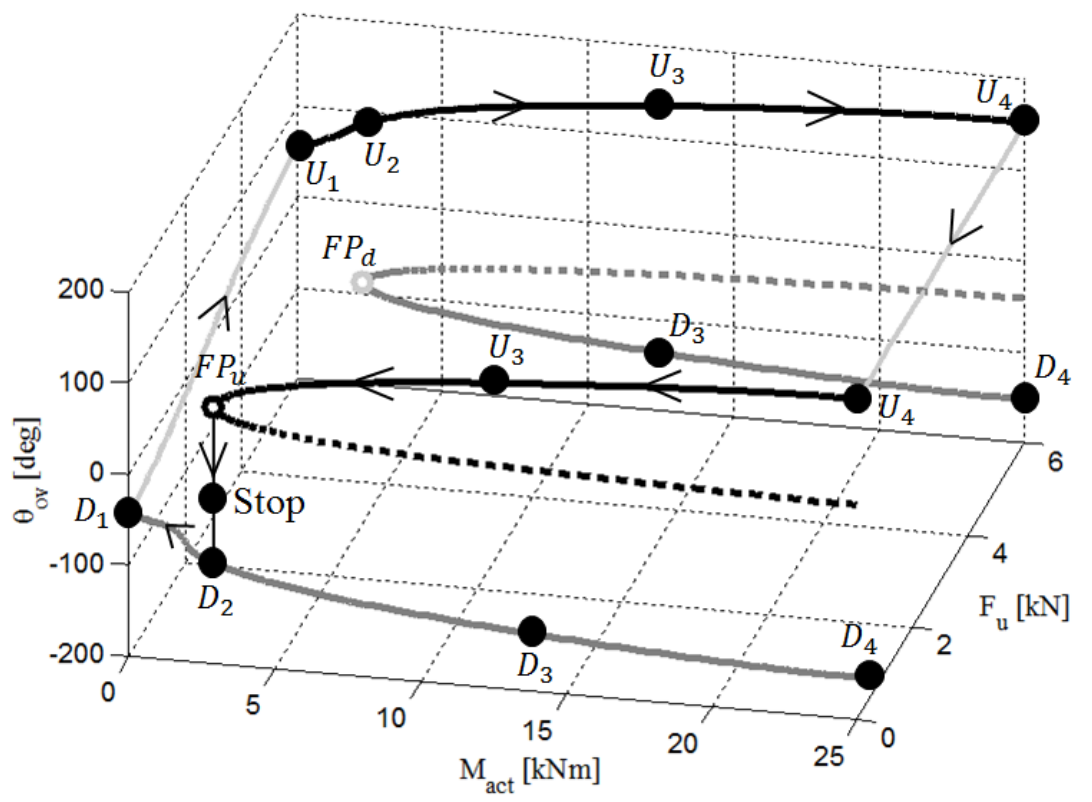

Fig.5 Numerical continuation results of a typical retraction and extension cycle

Combined with the schedules of landing gear retraction and extension, the retraction process can be inferred from the equilibrium solution curves. With reference to Fig 4, the single arrows on the curves denote the direction of the MLG's motion. In the unlock stage, the MLG moves from the starting position $\mathrm{D}_{1}\left(F_{u}=0 k N\right)$ to the unlocked position $\mathrm{U}_{1}\left(F_{u}=6 k N\right)$ driven by the unlock actuator. After that, the main strut retracts smoothly from position $\mathrm{U}_{1}$ to position $\mathrm{U}_{4}$ as the retraction actuator moment increases. Once the MLG has been locked at the stowed position $\mathrm{U}_{4}$, the unlock force could be released shown as the light grey curve, and the main strut stay at the same position $\mathrm{U}_{4}$ during this process. It can be found that, removing the unlock force has little effect on the MLG's position, with the landing gear staying at point $\mathrm{U}_{4}$. In addition, an inference can be made that the unlock actuator can be switched off with no influence once the retraction actuator generates sufficient force to counteract the external loads. 
For the extension stage, the MLG follows the black stable curve until the bifurcation point $F P_{u}$ with the help of the resistive moment supplied by the retraction actuator. Rather than following the unstable equilibrium, the trajectory jumps from the stable branch of the above-over-center curve to the stable branch of the below-over-center curve and reaches position $\mathrm{D}_{2}$. After that decreasing the retraction actuator force causes the MLG to go back to the initial position $D_{1}$. In reality this jump to $D_{2}$ is not completed due to stops, which are used to prevent the lock-links from folding downwards beyond a certain limit locking them together. Hence the equilibrium solution of the retraction motion on the dark grey curve would not be physically realizable in an actual MLG mechanism; instead, the mechanism hits the lock-link stops, and locks at the point labeled stop.

Contrary to the MLG's response with unlock force $F_{u}=6 \mathrm{kN}$, only one stable equilibrium solution $\mathrm{D}_{1}$ at $M_{a c t}=0 \mathrm{kNm}$ can be seen on the dark grey curve in the case of $F_{u}=0 \mathrm{kN}$. Increasing the retraction actuator moment causes the MLG to follow the stable below-over-center curve with the lock-links folding downwards and reach the retracted state $\mathrm{D}_{4}$ finally. The trajectory described by the dark grey curves would also not be physically realizable in reality due to the stops.

For clarity, all the MLG positions corresponding to the typical equilibrium points $\left(\mathrm{U}_{1}-\mathrm{U}_{4}\right.$ above-over-center) and $\left(D_{1}-D_{4}\right.$ below-over-center) are shown in Fig. 6, panels $\left(U_{1}-U_{4}\right)$ and $\left(D_{1}-D_{4}\right)$.

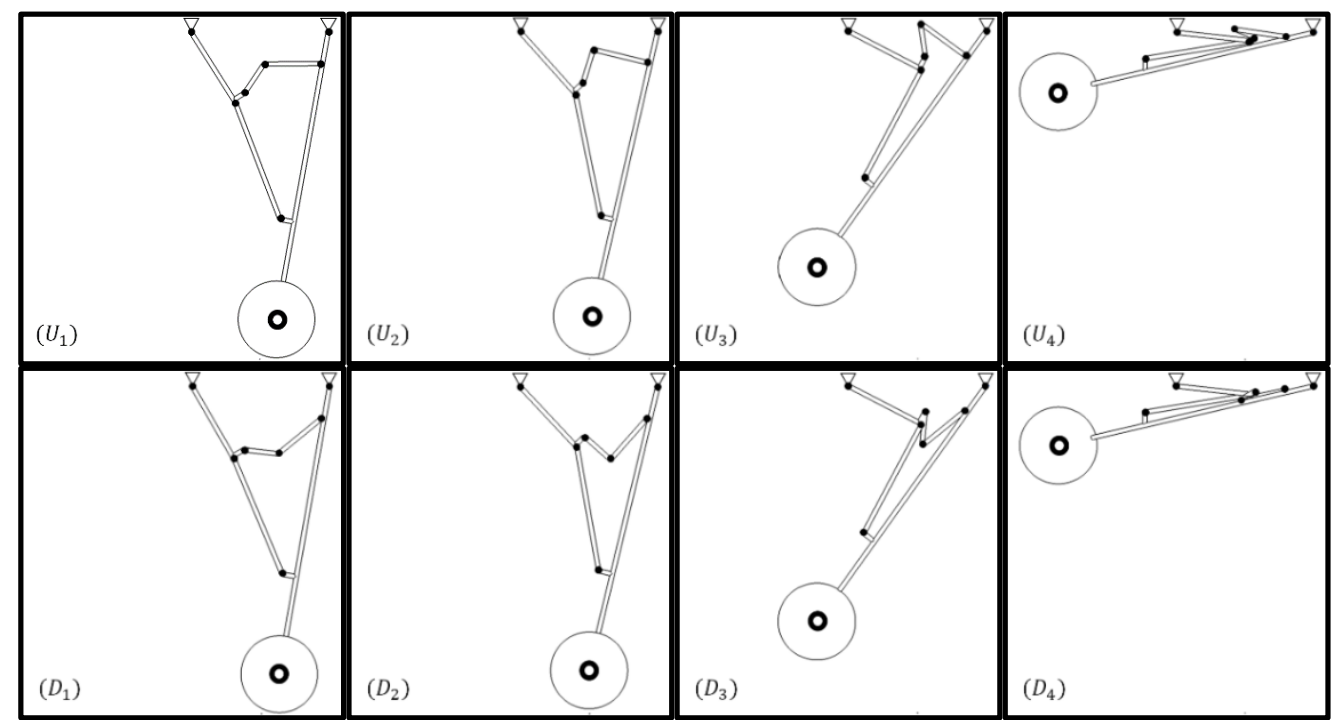

Fig. 6 Diagrams of the MLG retracts as the lock-links folds upwards $\left(U_{1}-U_{4}\right)$ and downwards $\left(D_{1}-D_{4}\right)$ 


\section{Effect of the unlock force on the MLG retraction cycle}

Discussions in the previous sections have shown that variation of unlock force could change the folding direction and the FP bifurcation's position of the lock-links, which represents the changing of some key properties of the MLG mechanism. As the focus of this section is to investigate the critical unlock force, under which the landing gear could retract normally with the lock-links folding upward, the effects of different unlock forces on the behavior of the MLG will be discussed in detail.

\section{A. Changes of the MLG Retraction Cycle with Different Unlock Force}

The equilibrium solutions of the retraction mechanism under 6 different unlock force cases are shown in Fig. 7, with the figures (a) and (f) corresponding to the behaviors shown in Fig. 6. The solid curves indicate stable solutions; dashed curves indicate unstable solutions. The black curve shows solutions where the lock-links are above-overcenter, whilst the grey curve denotes solutions where the lock-links are below-over-center. Single arrows indicate the direction of motion for an increasing retraction actuator moment starting from $M_{a c t}=0 \mathrm{kN} \cdot \mathrm{m}$. Fold point bifurcations are indicated by points $F P_{u}$ and $F P_{d}$. For clarity, the results are discussed in order of decreasing unlock force.

As shown in Fig. 7(a), the MLG can be unlocked with the unlock force $F_{u}=6 k N$ and retracted along the black stable above-over-center curve as the retraction moment increase. Compared with the results in Fig. 7(a), the retraction trajectory with the unlock force $F_{u}=5 \mathrm{kN}$ shown in Fig. 7(b) changes little except the beginning phase of the retraction trajectory, where the gradient of the curve has increased. Decreasing the unlock force to $F_{u}=3.5 \mathrm{kN}$, as shown in Fig. 7(c), a single stable equilibrium point at $M_{a c t}=0 \mathrm{kN} \cdot \mathrm{m}$ can still be found on the stable above-overcenter curve, which means the MLG could be retracted by the retraction actuator. However, unlike the stable curves in Fig. 7(a) and Fig. 7(b), two fold bifurcation points $F P_{u}^{1}$ and $F P_{u}^{2}$ appear and divide the stable above-over-center curve into two stable parts (upper stable branch and lower stable branch), which can form a hysteresis loop shown in Fig. 7(c). Before reaching the bifurcation $F P_{u}^{1}$, the MLG follows the lower black stable branch as the retraction moment increases, but small changes of over-center angle indicate the MLG hardly moves while on the lower

branch. Increasing the retraction moment past $F P_{u}^{1}$, the retraction trajectory jumps from the lower stable branch to 
the upper stable branch, which means the lock-links fold sharply, and the MLG gets into the regular retraction curve. The reason for the two bifurcation points and the resulting hysteresis loop is that the MLG stays at the critical state between unlocked and locked states. In other words, the MLG is incompletely unlocked with the unlock force of $F_{u}=3.5 \mathrm{kN}$ until the jump where the links "snap" unlocked. Fig. 8(d) shows the equilibrium solution with the unlock force of $F_{u}=2.5 \mathrm{kN}$. In this case, the MLG can only follow the light grey stable curve between the aboveover-center and below-over-center curves. As shown in the Fig. 8(d), increasing retraction moment hardly moves the mechanism, which means the MLG could not be unlocked when the unlock force $F_{u}=2.5 \mathrm{kN}$.

Regarding the results shown in Fig. 7(e) and Fig. 7(f), the conclusions are similar with the ones shown in Fig. 7(c) and Fig. 7(b) respectively, with the only difference of the lock-links' folding direction. To be specific, two bifurcation points occur on the below-over-center curve and cause the forming of hysteresis loop in the case of $F_{u}=1.5 \mathrm{kN}$ (as in Fig. 7(e)). When the unlock force reduces to zero, the MLG can be retracted with the lock-links folding downwards.
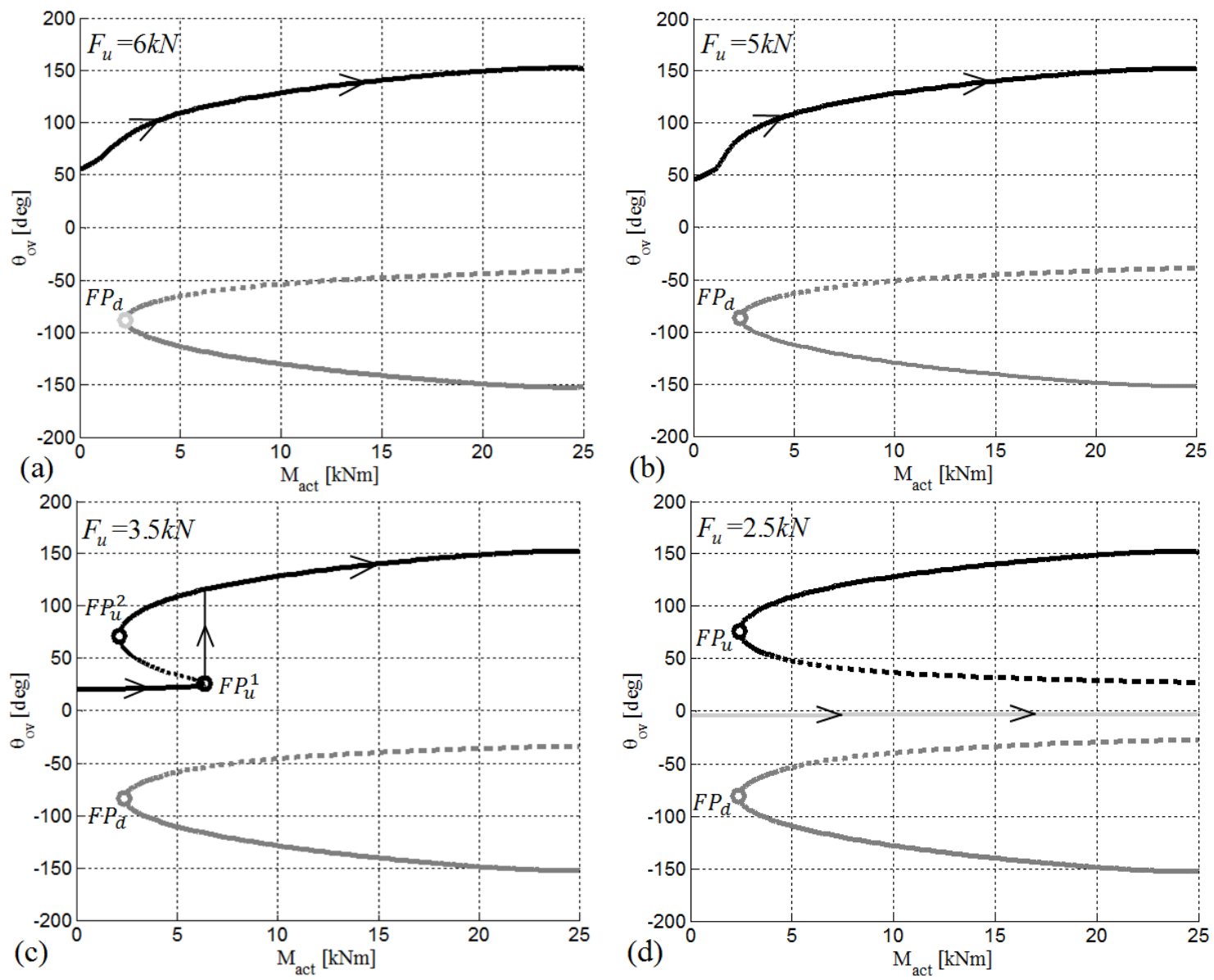

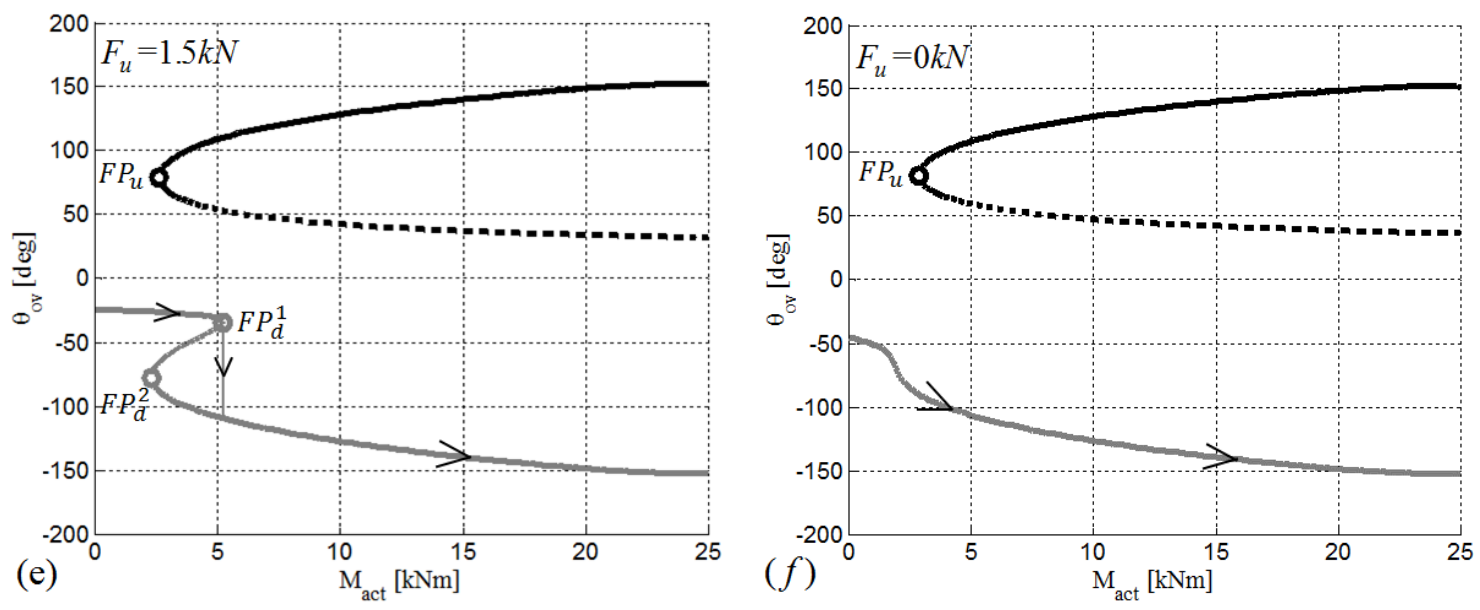

Fig 7 Numerical continuation results under different unlock actuator force

\section{B. The Definition of Different Unlock Region and Critical Unlock Positions}

Based on the above analysis, the effect of unlock force on the MLG's retraction behavior can be divided to five regions, which are shown in Fig. 8:

(1) The above-over-center, completely unlocked region: in this region, the MLG has been unlocked and can be retracted smoothly by the retraction actuator.

(2) The above-over-center, incompletely unlocked region: in this region, the MLG will go through a gentle-slope trajectory, and will only retract smoothly once it jumps past the bifurcation point.

(3) The locked region: in this region, the MLG can never be retracted by the retraction actuator.

(4) The below-over-center, incompletely unlocked region: in this region, the MLG will go through a gentle-slope trajectory, and will only retract smoothly once it jumps past the bifurcation point. However, the motion that the locklinks fold downwards would not happen in reality owing to the stops.

(5) The below-over-center, completely unlocked region: in this region, the MLG could be retraced by the retraction actuator with the lock-links folding downward. Again, this is not physically realizable in an actual retraction mechanism due to the lock-links stops. 

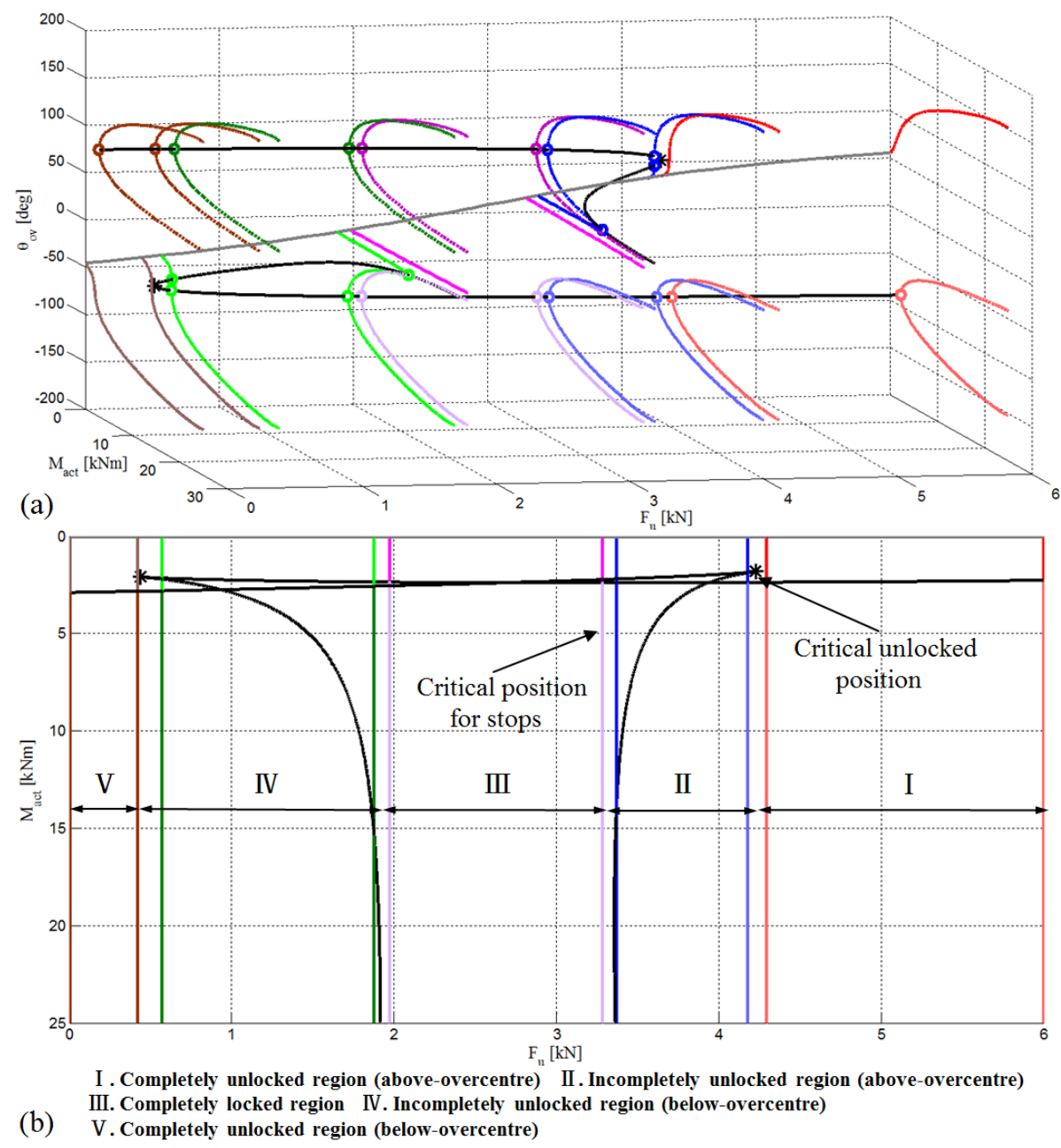

\section{Fig. 8 Bifurcation diagrams and projection of the MLG retraction cycle with different unlock forces}

As shown in Fig. 8(b), two important definition of critical positions are provided in this section: one is the critical position for the design of the lock-links' stops; the other is the critical unlocked position which means the MLG has been completely unlocked.

Stops can be defined as the locking point where the lock-link stops contact with each other to prevent the locklinks folding downwards. The locking point is a position where the MLG is totally locked, thus the locking point should locate in the completely locked region or below this region (with the stops locking the mechanism). As a result, the critical position for the design of the lock-links' stops is the upper boundary of the completely locked region shown in Fig. 8(b).

The unlocked state indicates where the MLG can be retracted smoothly with the retraction actuator, and the critical unlocked position indicates the interface between the above-over-center, completely unlocked and the aboveover-center, incompletely unlocked regions. From the locus of the FPs as a function of unlock force $F_{u}$ and 
retraction moment $M_{a c t}$ shown in Fig. 8(b), it can be seen that the two fold bifurcation points come together and disappear at a cusp point (indicated by *) as the unlock force $F_{u}$ increases. The disappearance of the FP means the retraction mechanism enters the completely unlocked region. Therefore, the position corresponding to the cusp point is the critical unlocked position and the unlock force corresponding to the cusp point is the critical unlock force representing the minimum force that should be used to unlock the mechanism.

\section{The Transition Between Different Unlock Regions}

Firstly, consider the transition between the above-over-center, completely unlocked and the above-over-center, incompletely unlocked regions which captures the critical force required for unlocking: In the above-over-center, incompletely unlocked region, two fold bifurcation points appear on the above-over-center retraction trajectory. From the detailed graphs of the locus of the FPs shown in Fig. 9, it can be seen that the two fold bifurcation points come together and disappear at a cusp point as the unlock force $F_{u}$ increases. The disappearance of FP means the incompletely unlocked region transfers to the completely unlocked region.
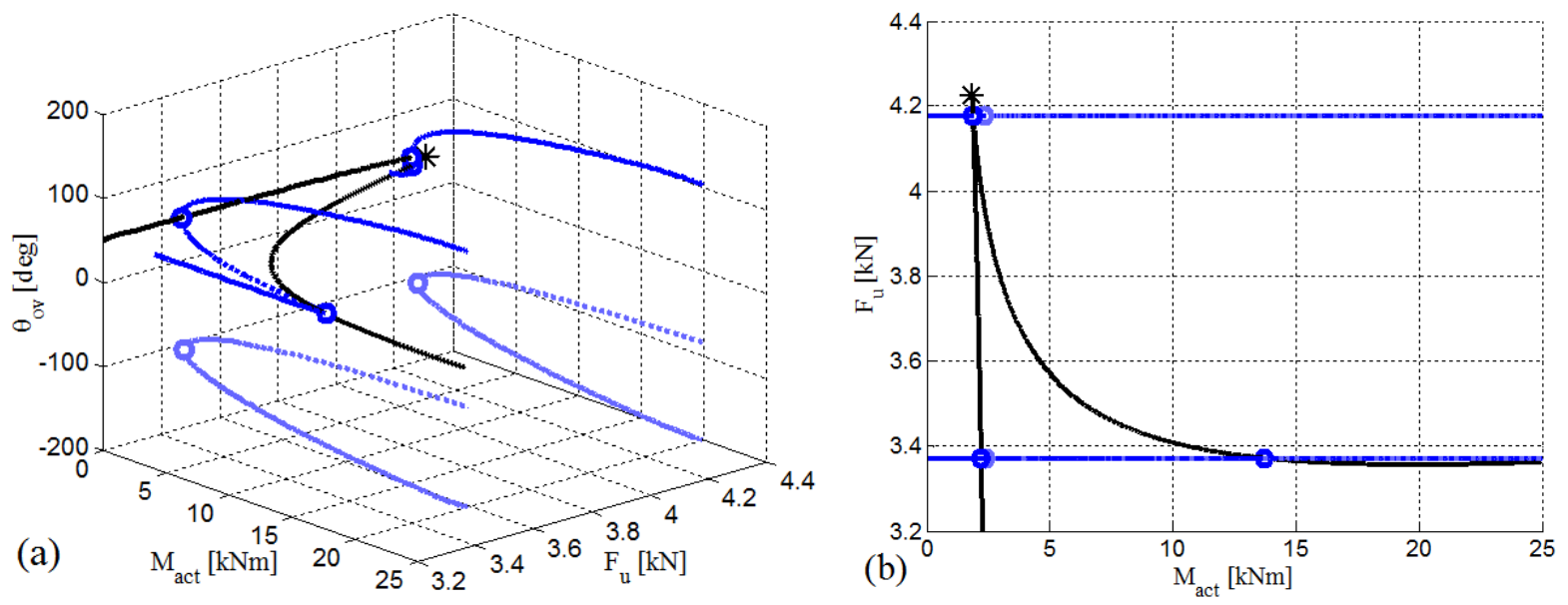

Fig. 9 Fold bifurcation point trace varied with unlock forces $\boldsymbol{F}_{\boldsymbol{u}}$ on the above-over-center branch

Secondly, consider the transformation between the above-over-center, incompletely unlocked and the completely locked regions which captures the critical position for the stops: In the above-over-center, incompletely unlocked region, the MLG can be retracted on the condition that the retraction moment passes the bifurcation point $F P_{u}^{1}$, namely, the critical value. As shown in Fig. 8(b), the locus of bifurcation points in the above-over-center, incompletely unlocked region shows the $F P_{u}^{1}$ arbitrarily close to the upper boundary of completely locked region and the corresponding moment tending to infinity. That is to say, if the unlock force is located in the completely locked 
region, the critical moment corresponding to $F P_{u}^{1}$ is very large and requiring an unrealistic level of power from the hydraulic system, thus the landing gear could not retract in this region.

\section{Optimization of Critical Unlock Force Considering Multiple Geometric Parameters}

The conclusions in section 4 show that the unlock actuator needs to provide sufficient force to drive the locklinks past the critical angle before the MLG can be retracted smoothly, which indicates that the unlock force has a great effect on the landing gear's retraction performance. Additionally, previous work [2] has shown that the unlock force is affected by geometry parameters, such as the spring stiffness $k$, the unstrained spring length $l_{u}$ and the attachment point of spring $s_{l}$. Therefore, in this section, the dependence of the critical unlock angle and the unlock force on different parameters of interest is analyzed in detail. Following this, an optimization study is carried out to reduce the force needed to unlock the mechanism.

\section{A. Effect of Multiple Parameters on the Critical Unlock Angle}

Before analyzing the effect of multiple parameters on the unlock force, the variation of critical unlock angle due to changes in gear parameters is considered first. It should be noted that three key parameters are of interest: including spring stiffness $k$; unstrained spring length $l_{u}$; attachment point $s_{l}$. The parameter values of three different test cases are shown in Table 2.

Table 2 Geometry parameters values of different cases

\begin{tabular}{cccc}
\hline \hline Case number & Spring stiffness $k$ & Unstrained length $l_{u}$ & Attachment point $s_{l}$ \\
\hline 1 & $2 \mathrm{kN} / \mathrm{m}$ & $0.34 \mathrm{~m}$ & $0.05 \mathrm{~m}$ \\
2 & $4 \mathrm{kN} / \mathrm{m}$ & $0.365 \mathrm{~m}$ & $0.1 \mathrm{~m}$ \\
3 & $6 \mathrm{kN} / \mathrm{m}$ & $0.39 \mathrm{~m}$ & $0.15 \mathrm{~m}$ \\
\hline \hline
\end{tabular}

Bifurcation diagrams in the above-over-center, incompletely unlocked region are shown in Fig. 10. For the different cases, thin solid curves indicate the unlock process; dashed curves indicate the locus of FPs in the aboveover-center, incompletely unlocked region; heavy solid curves indicate the over-center angle varied with retraction moment.

The unlock curve, represented by the thin solid curves, moves down as the parameters are changed, which means the required unlock force corresponding to the same unlock angle increases. As suggested by the variation in the dashed curves, the unlock force corresponding to the unlock cusp point, namely critical unlock force, increases with 
the three key parameters increasing. However, the critical unlock angles corresponding to the cusp points, represented by black filled circles, do not change with parameters of interest. The results demonstrate that the three key parameters $\left(k, l_{u}, s_{l}\right)$ may change the critical unlock force, but they have little effect on the critical angle which is related only to the structural parameters of the landing gear links.
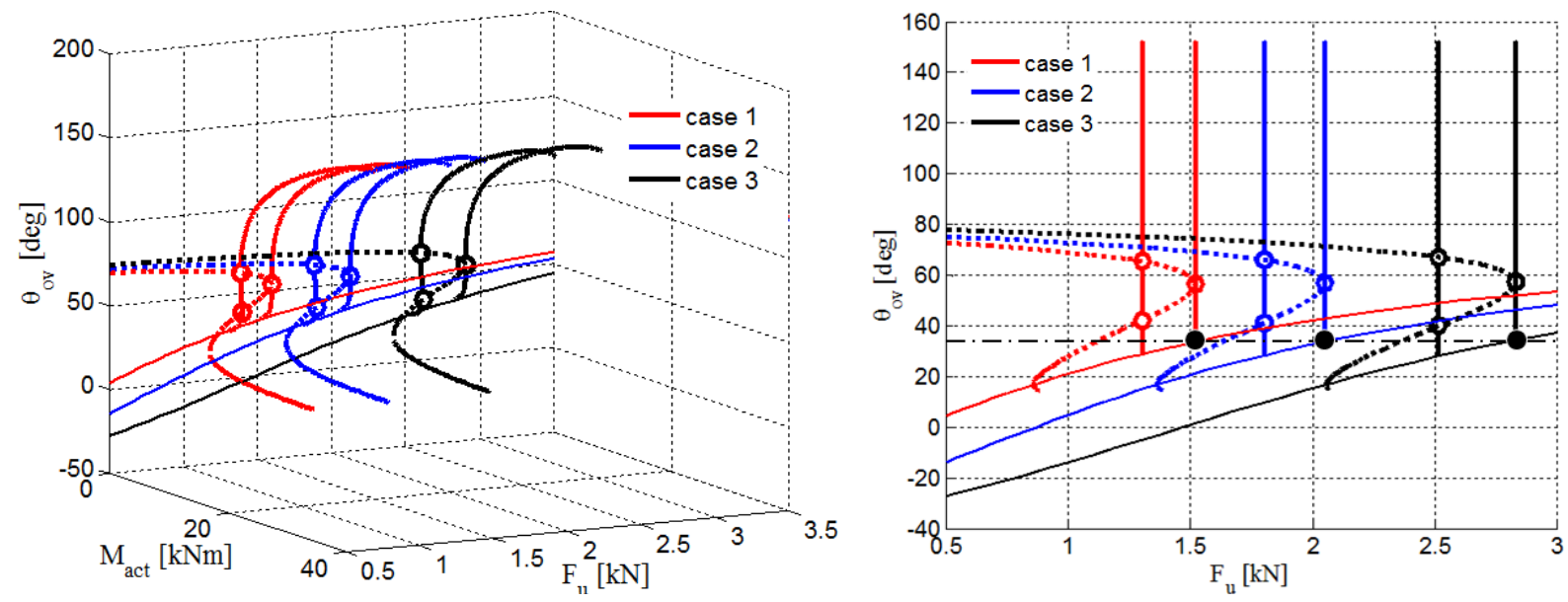

Fig. 10 Numerical continuation results on the space of $k, l_{u}$ and $s_{\mathbf{l}}$.

\section{B. Effect of Multiple Parameters on the Unlock Force}

This section demonstrates how the unlock force varies with the key parameters of interest. Taking the horizontal state of the lock-links as an example to investigate how all the parameters $\left(k, l_{u}, s_{l}\right)$ affect the unlock force $F_{u}$ [2], the following results can be obtained by using $\theta_{o v}$ as a fixed parameter $\left(\theta_{o v}=0 \mathrm{deg}\right)$ and setting unlock actuator force $F_{u}$ as the state variable.
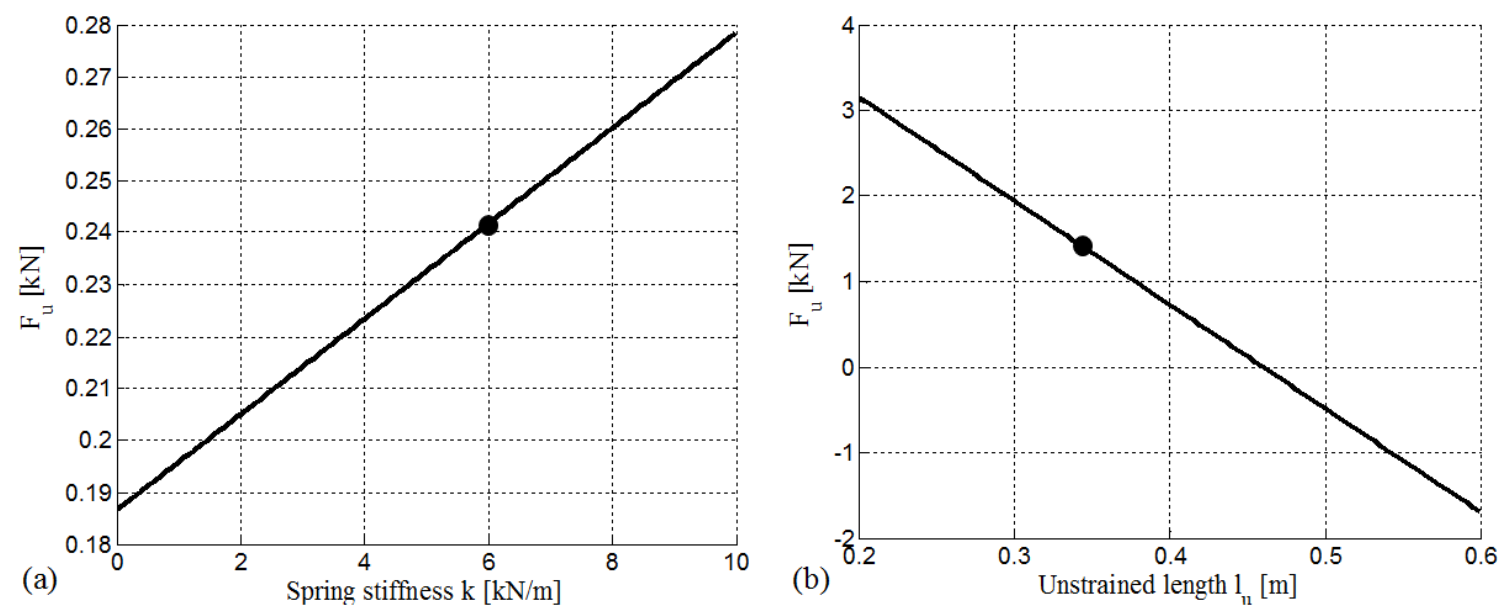


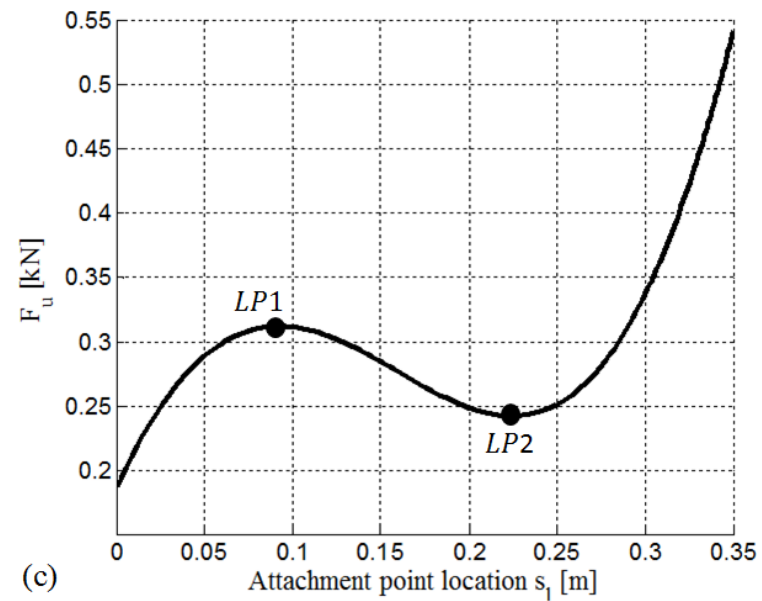

Fig. $11 F_{u}$ varies with three key geometric parameters

As shown in Fig. 11(a) and Fig. 11(b), the spring stiffness $k$ and unstrained length $l_{u}$ both have linear effects on the unlock force $F_{u}$. The increase of spring stiffness $k$ and the decrease of unstrained length $l_{u}$ increase the moment created by the spring force about the lock-link-side-stay joint, resulting in an increase of the required unlock force.

The relationship between the unlock force and the position of the spring attachment point, shown in Fig. 11(c), is nonlinear and two LPs appear at the peak and though of the curve. To be specific, the trend of unlock force as a function of unstrained spring length can be divided into three parts: first it increases, then it decreases, and finally it increases again. The reason for this trend can be explained with Fig. 12 as follows. The required unlock force to maintain equilibrium changes with the moment produced by the spring force, which is related to the attachment point $s_{l}$. The increase of $s_{l}$ causes the distance between the spring ends and spring force to firstly decrease and then increase. In contrast to this, the moment arm of spring firstly increases and then decreases with the increase of $s_{l}$. Thus the spring length and the moment arm of spring make up a pair of conflicting factors, and the weight change of the effect on unlock force between the two conflicting factors leads to the nonlinear relationship shown in Fig. 11(c).

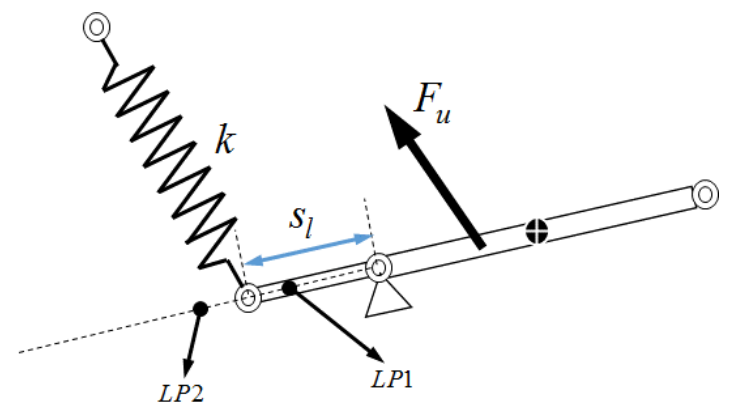

Fig 12 Force diagram of lock spring 


\section{Optimization of the Unlock Force}

In this section, an optimization is carried out to minimize the critical unlock force through selection of three design variables: spring stiffness $k$; unstrained spring length $l_{u}$ and spring attachment point $s_{l}$. The Multi-Island Genetic Algorithm (MIGA) method [21] is adopted to optimize the unlock force based on a Computer Aided Engineering (CAE) software Isight, combined with MATLAB. Isight is a Process Integration and Design Optimization (PIDO) software framework [22], which enables various applications to be easily integrated including MATLAB.

The process of optimization is illustrated by the flow chart shown in Fig. 13. The framework consists of Isight and $\mathrm{COCO}$, which are used as a data processing toolbox and a continuation algorithm toolbox respectively. At the beginning of each iteration, a selection of $k, l_{u}, s_{l}$ based on the rules of MIGA is conducted by Isight and transferred to COCO. By using numerical continuation algorithm, the critical unlock force (object variable) $F_{u}^{c}$ and the initial over-center angle $\theta_{o v}^{0}$ can be obtained and transferred back to Isight for checking the convergence property of object variable. After that, the iterative computation is performed again until the convergence property meets the requirements. The target of continuation algorithm is to obtain the cusp point in the above-over-center, incompletely unlocked region, which should be found exactly under a specific set of parameters $\left(k, l_{u}, s_{l}\right)$. Based on the above analysis, it can be seen that $k, l_{u}, s_{l}$ affect the unlock force $F_{u}$ in the above-over-center, incompletely unlocked region, but have little effect on the over-center angle $\theta_{o v}$. Thus the location of above-over-center, incompletely unlocked region can be found based on the over-center angle $\theta_{o v}$. According to the details shown in Fig. 10, the landing gear will operate in the above-over-center, incompletely unlocked region within the scope of over-center angle $\theta_{o v}=\left(20^{\circ} \sim 33.6^{\circ}\right)$, and two fold bifurcation points appear on the retraction trajectory.

Thus the flow of each iteration can be indicated as follows: firstly, calculate the unlock curve by using continuation algorithm and choose $\theta_{o v}=25^{\circ}$ as the start point where the landing gear is in the incompletely unlocked state. Secondly, two 1-parameter continuation slices (2 FPs) can be obtained by increasing the retraction moment. Finally, 2-parameters continuation is performed to get the locus of FPs and the cusp point. The unlock force corresponding to the cusp point is the critical unlock force $F_{u}^{c}$. 


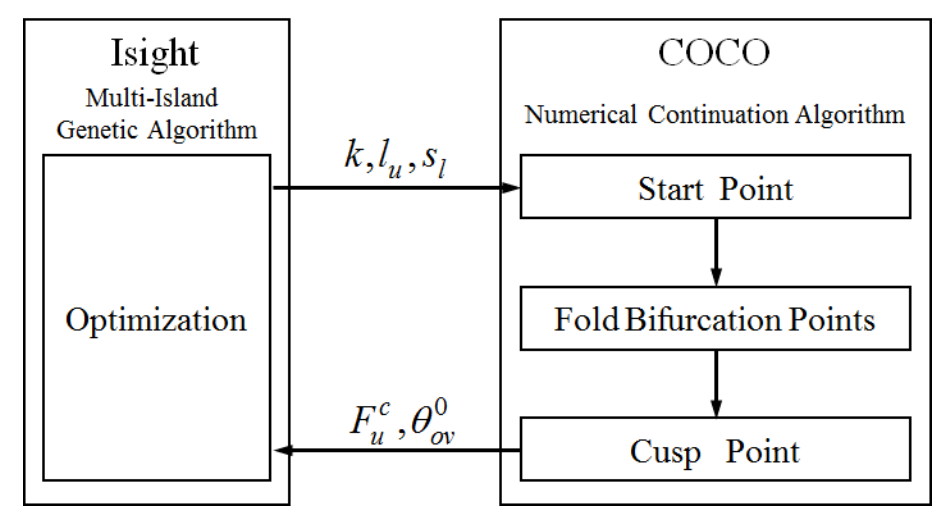

Fig. 13 Flow chart of optimization combined with continuation algorithm

The bounds of variables $\mathbf{X}=\left[k, l_{u}, s_{l}\right]$ are set as $\mathbf{X}_{l b}=[2 K N / m, 0.2 m, 0.05 m]$ and $\mathbf{X}_{u b}=[8 K N / m, 0.5 m, 0.4 m]$.

Additionally, the constraint of initial over-center angle $\left(\theta_{o v}^{0}=-45.0 \mathrm{deg}\right)$ needs to be included in the optimization in order to guarantee the locking mechanism's ability to lock. The objective variables should not only realize the minimum critical unlock force, but also make the lock-links reach the initial over-center angle, which needs to be the same as the initial over-center angle realized by the default parameters with no stops.

Table 3 Optimization results for minimizing critical unlock force

\begin{tabular}{cccccc}
\hline \hline & $\begin{array}{c}\text { Spring stiffness } \\
k\end{array}$ & $\begin{array}{c}\text { Unstrained } \\
\text { length } l_{u}\end{array}$ & $\begin{array}{c}\text { Attachment } \\
\text { point } s_{l}\end{array}$ & $\begin{array}{c}\text { Lock ability } \\
\text { Initial angle } \theta_{o v}^{0}\end{array}$ & $\begin{array}{c}\text { Critical unlock } \\
\text { force } F_{u}^{c}\end{array}$ \\
\hline Default & $6 \mathrm{kN} / \mathrm{m}$ & $0.34 \mathrm{~m}$ & $0.225 \mathrm{~m}$ & $-45.0 \mathrm{deg}$ & $4.227 \mathrm{kN}$ \\
Optimization & $4.82 \mathrm{kN} / \mathrm{m}$ & $0.25 \mathrm{~m}$ & $0.102 \mathrm{~m}$ & $-45.2 \mathrm{deg}$ & $2.664 \mathrm{kN}$ \\
\hline
\end{tabular}
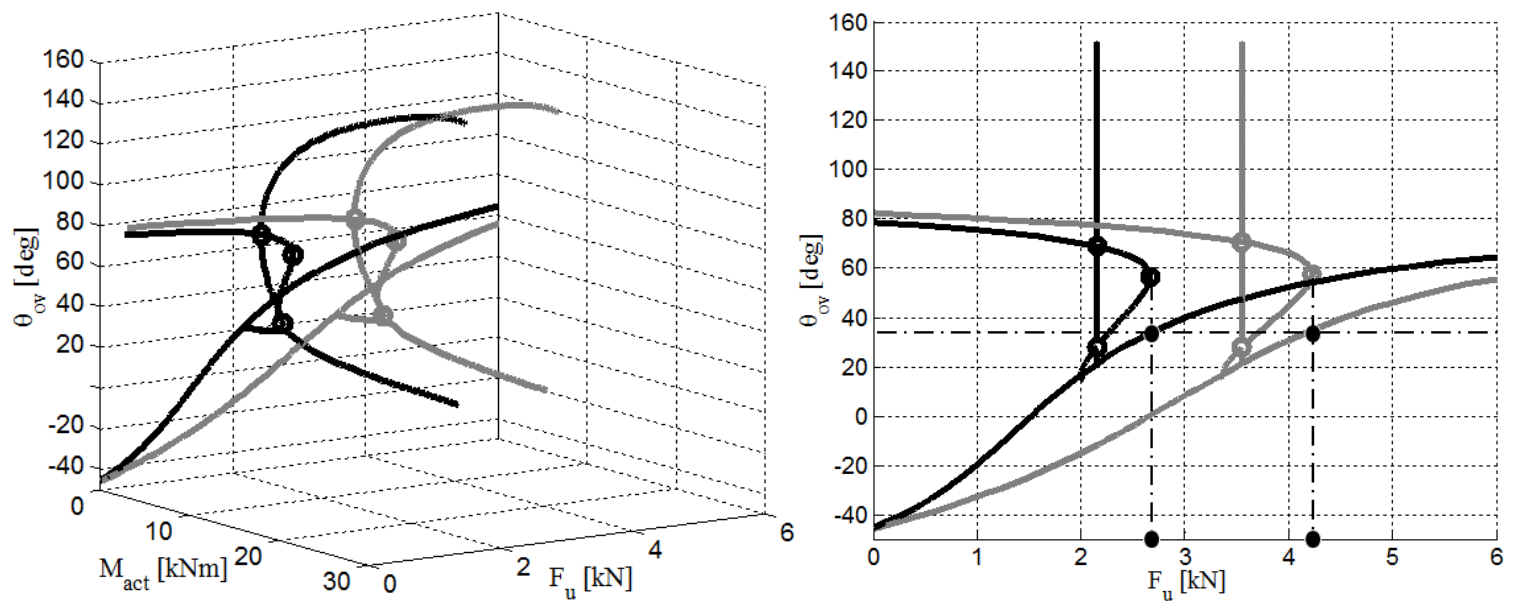

Fig. 14 Optimization results of critical unlock force 
The optimization results are shown in Fig. 14, with numerical results given in Table 3. The results show that, the optimized parameters make the critical unlock force decrease greatly by $37 \%$ while ensuring the same initial overcenter angle of the lock-links with no stops.

\section{Conclusion}

With numerical continuation, the effects of unlock force and parameters of interest on a MLG's retraction performance can be analyzed efficiently. The unlock force has a great effect on the locking mechanism: the main strut can be retracted smoothly only when the lock-links pass a critical angle. For different unlock forces, unlock states of the MLG can be divided into five separate regions. Two important critical positions are identified through bifurcation analysis method and can be described as follows: the critical position for the lock-links' stops is the upper boundary of the completely locked region; the critical unlocked position locates at the interface between the above-over-center, completely unlocked and the above-over-center, incompletely unlocked regions. Analyzing the effects of key parameters on locking mechanism shows that spring parameters do not affect the critical unlock overcenter angle, but do have effect on the critical unlock force. The spring stiffness $k$ and unstrained length $l_{u}$ both

have linear effects, while the attachment point $s_{l}$ has nonlinear effect, on the unlock force $F_{u}$. Based on optimization of unlock force, an appropriate set of spring parameters can be obtained to make a $37 \%$ reduction on unlock force $F_{u}$, whilst maintaining the same level of locking ability, with an initial over-center angle of $\theta_{o v}^{0}=-45.2 \mathrm{deg}$. With regards to future work, this technique for optimization of critical unlock force could be applied to nose landing gear (NLG) which use the same locking mechanism to down-lock and up-lock the strut [2, 20]. The optimization design of the NLG's locking spring should be more complex since it is a multi-objective and multi-constrained optimization problem. Three optimization objects would be considered: the minimized critical unlock force for both the up-lock and down-lock mechanism; the minimized spring stiffness to provide sufficient force to up-lock the NLG in the stowed position.

\section{Acknowledgments}

This work was financially supported by the National Natural Science Foundation of China (11372129), the Priority Academic Program Development of Jiangsu Higher Education Institutes, and the China Scholarship Council Studentship. In additions S. A. N. was supported by an EPSRC fellowship (EP/K005375/1). 


\section{Appendix}

Based on the description given in section II.E, the internal forces may be derived from the actuator forces and landing gear position using the matrix expression $A \bar{F}-\bar{B}=0 \quad$ (Eq. 14) where

$$
\begin{aligned}
& A=\left[\begin{array}{cccccccccccccc}
0 & C_{1} & C_{2} & -l_{2} s \theta_{2} & l_{2} c \theta_{2} & 0 & 0 & 0 & 0 & 0 & 0 & 0 & 0 & C_{3} \\
0 & 0 & 0 & -1 & 0 & 0 & 1 & 0 & 0 & 0 & 0 & 0 & 0 & 0 \\
0 & 0 & 0 & 0 & -1 & 0 & 0 & 1 & 0 & 0 & 0 & 0 & 0 & 0 \\
0 & 0 & 0 & 0 & 0 & 0 & -l_{3} s \theta_{3} & l_{3} c \theta_{3} & 0 & 0 & 0 & 0 & 0 & 0 \\
0 & -1 & 0 & 0 & 0 & 0 & 0 & 0 & 1 & 0 & 0 & 0 & 0 & \eta_{y} \\
0 & 0 & -1 & 0 & 0 & 0 & 0 & 0 & 0 & 1 & 0 & 0 & 0 & \eta_{z} \\
0 & 0 & 0 & 0 & 0 & 0 & 0 & 0 & l_{4} s \theta_{4} & -l_{4} c \theta_{4} & 0 & 0 & 0 & C_{4} \\
0 & 0 & 0 & 0 & 0 & 0 & 0 & 0 & -1 & 0 & 0 & 1 & 0 & 0 \\
0 & 0 & 0 & 0 & 0 & 0 & 0 & 0 & 0 & -1 & 0 & 0 & 1 & 0 \\
0 & 0 & 0 & 0 & 0 & 0 & 0 & 0 & 0 & 0 & 0 & l_{5} s \theta_{5} & -l_{5} c \theta_{5} & 0 \\
1 & 0 & 0 & 0 & 0 & 1 & 0 & 0 & 0 & 0 & 1 & 0 & 0 & 0 \\
l_{O B ; A} & 0 & 0 & 0 & 0 & 0 & 0 & 0 & 0 & 0 & 0 & 0 & 0 & 0 \\
0 & 0 & 0 & 0 & 0 & C_{5} & 0 & 0 & 0 & 0 & C_{6} & 0 & 0 & 0 \\
0 & 0 & 0 & 0 & 0 & C_{7} & C_{8} & C_{9} & 0 & 0 & C_{10} & C_{11} & C_{12} & 0
\end{array}\right],
\end{aligned}
$$

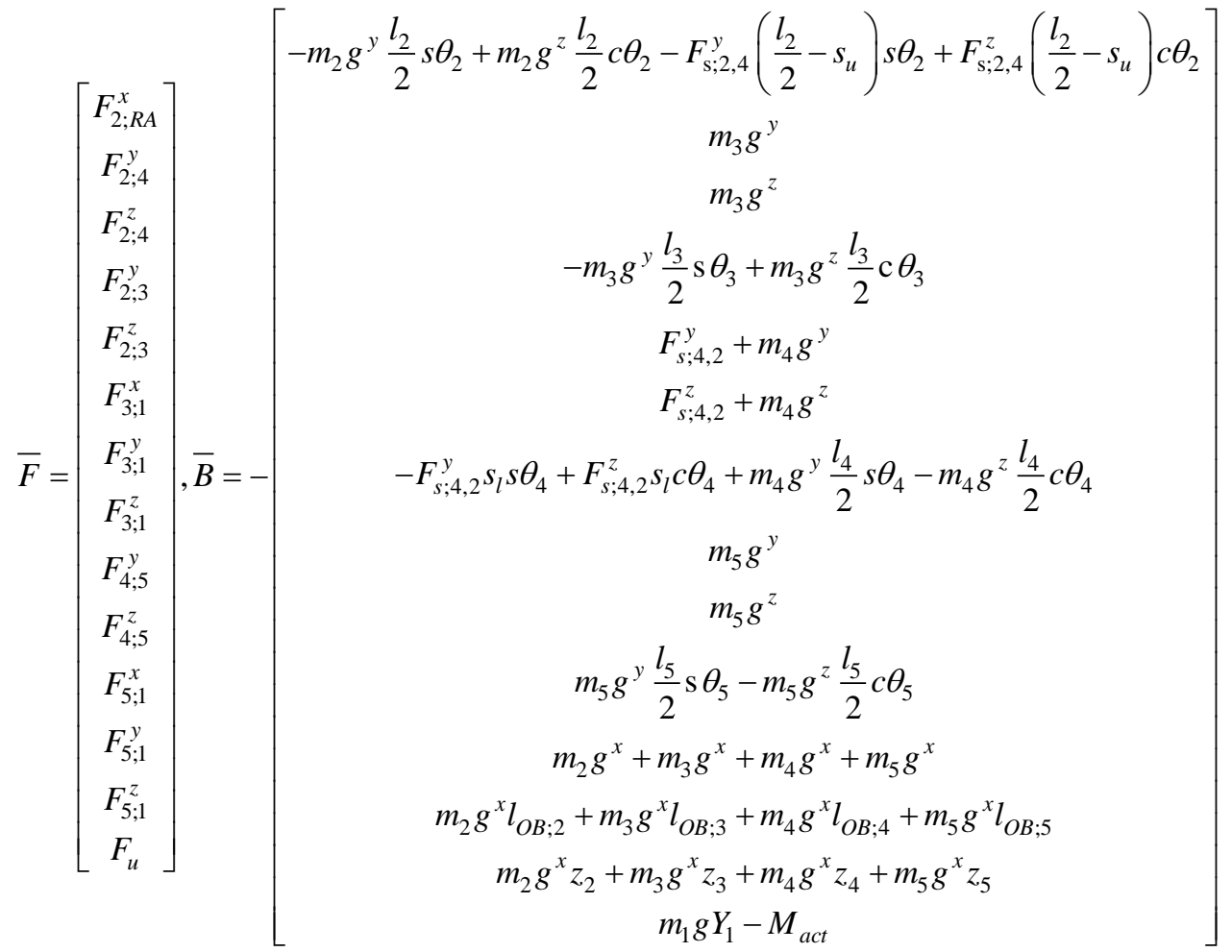

and the shorthand $s_{i}=\sin _{i}, c_{i}=\cos { }_{i}$ for $i=1,2,3,4,5$ has been used along with $C_{1}=-l_{24} \cdot \sin \left(\beta_{24}+\theta_{2}\right)-\frac{l_{2}}{2} \cdot \sin \theta_{2} C_{2}=l_{24} \cdot \cos \left(\beta_{24}+\theta_{2}\right)+\frac{l_{2}}{2} \cdot \cos \theta_{2}$ 


$$
\begin{aligned}
& C_{3}=-\eta_{y} \cdot\left(\frac{l_{2}}{2}-u n\right) \cdot \sin \theta_{2}+\eta_{z} \cdot\left(\frac{l_{2}}{2}-u n\right) \cdot \cos \theta_{2} C_{4}=\eta_{y} \cdot\left(\frac{l_{4}}{2}-u m\right) \cdot \sin \theta_{4}-\eta_{z} \cdot\left(\frac{l_{4}}{2}-u m\right) \cdot \cos \theta_{4} \\
& C_{5}=z_{3}+\frac{l_{3}}{2} \cdot \sin \theta_{3} C_{6}=z_{5}-\frac{l_{5}}{2} \cdot \sin \theta_{5} C_{7}=t_{2,1} \cdot B_{Z}-t_{3,1} \cdot B_{Y} C_{8}=t_{2,2} \cdot B_{Z}-t_{3,2} \cdot B_{Y} \\
& C_{9}=t_{2,3} \cdot B_{Z}-t_{3,3} \cdot B_{Y} C_{10}=t_{2,1} \cdot F_{Z}-t_{3,1} \cdot F_{Y} C_{11}=t_{2,2} \cdot F_{Z}-t_{3,2} \cdot F_{Y} C_{12}=t_{2,3} \cdot F_{Z}-t_{3,3} \cdot F_{Y}
\end{aligned}
$$

\section{References}

1. Curry, N. S. Aircraft Landing gear Design: Principle and Practice. Washington, D.C.: AIAA, 1988.

2. Knowles, J. A. C., Krauskopf, B., and Lowenberg, M. H. "Numerical Continuation Applied to Landing Gear Mechanism Analysis," Journal of Aircraft Vol. 48, No. 4, 2011, pp. 1254-1262.

doi: $10.2514 / 1 . C 031247$

3. Wei, X. H., Yin, Y., Chen, H., and Nie, H. "Modeling and simulation of Aircraft Nose Landing Gear Emergency Lowering Using Co-simulation Method," Advances in Design Technology, Vols 1 and 2 Vol. 215-216, 2012, pp. 1213-1218.

doi: 10.4028/www.scientific.net/AMM.215-216.1213

4. Hać, M., and Form, K. "Design of Retraction Mechanism of Aircraft Landing Gear," Mechanics and Mechanical Engineering Vol. 12, No. 4, 2008, pp. 357-373.

5. Yin, Y., Nie, H., Ni, H. J., and Zhang, M. "Reliability Analysis of Landing Gear Retraction System Influenced by Multifactors," Journal of Aircraft Vol. 53, No. 3, 2016, pp. 713-724.

doi: $10.2514 / 1 . C 033333$

6. Zhang, H., Ning, J., and Schmelzer, O. "Integrated Landing Gear System Retraction/Extension Analysis Using ADAMS," International ADAMS User Conference. Orlando, FL, 2000.

7. Yin, Y., Nie, H., Wei, X. H., and Ni, H. J. "Retraction system performance analysis of landing gear with the influence of multiple factors," Journal of Beijing University of Aeronautics and Astronautics Vol. 41, No. 5, 2015, pp. 953-960.

8. Amirouche, F. M. L. Fundamentals of Multibody Dynamics: Theory and Applications. Chicago: Birkhäuser Basel, 2006.

9. Krauskopf, B., Osinga, H. M., and Galán-Vioque, J. Numerical Continuation Methods for Dynamical Systems. Netherlands: Springer, 2007. 
10. Knowles, J. A. C., Krauskopf, B., and Lowenberg, M. "Numerical continuation analysis of a threedimensional aircraft main landing gear mechanism," Nonlinear Dynamics Vol. 71, No. 1-2, 2013, pp. 331-352.

doi: $10.1007 / \mathrm{s} 11071-012-0664-\mathrm{z}$

11. Knowles, J. A. C., Krauskopf, B., Lowenberg, M. H., Neil, S. A., and Thota, P. "Numerical Continuation Analysis of a Dual-Sidestay Main Landing Gear Mechanism," Journal of Aircraft Vol. 51, No. 1, 2014, pp. 129-143.

doi: $10.2514 / 1 . C 032130$

12. Dankowicz, H., and Schilder, F. Recipes for Continuation. Philadelphia: Society for Industrial and Applied Mathematics, 2013

13. Sharma, S., Coetzee, E. B., Lowenberg, M. H., Neild, S. A., and Krauskopf, B. "Numerical continuation and bifurcation analysis in aircraft design: an industrial perspective," Philosophical Transactions of the Royal Society a-Mathematical Physical and Engineering Sciences Vol. 373, No. 2051, 2015.

doi: ARTN 20140406

10.1098/rsta.2014.0406

14. Thota, P., Krauskopf, B., and Lowenberg, M. "Multi-parameter bifurcation study of shimmy oscillations in a dual-wheel aircraft nose landing gear," Nonlinear Dynamics Vol. 70, No. 2, 2012, pp. 1675-1688. doi: $10.1007 / \mathrm{s} 11071-012-0565-1$

15. Howcroft, C., Krauskopf, B., Lowenberg, M. H., and Neild, S. A. "Influence of Variable Side-Stay Geometry on the Shimmy Dynamics of an Aircraft Dual-Wheel Main Landing Gear," Siam Journal on Applied Dynamical Systems Vol. 12, No. 3, 2013, pp. 1181-1209.

doi: $10.1137 / 120887643$

16. Feng, F., Nie, H., Zhang, M., and Peng, Y. M. "Effect of Torsional Damping on Aircraft Nose Landing-

Gear Shimmy," Journal of Aircraft Vol. 52, No. 2, 2015, pp. 561-568.

doi: $10.2514 / 1 . C 032772$

17. Rankin, J., Desroches, M., Krauskopf, B., and Lowenberg, M. "Canard cycles in aircraft ground dynamics," Nonlinear Dynamics Vol. 66, No. 4, 2011, pp. 681-688.

doi: 10.1007/s11071-010-9940-y 
18. Rankin, J., Krauskopf, B., Lowenberg, M., and Coetzee, E. "Nonlinear Analysis of Lateral Loading During Taxiway Turns," Journal of Guidance Control and Dynamics Vol. 33, No. 6, 2010, pp. 17081717.

doi: $10.2514 / 1.50626$

19. Rankin, J., Krauskopf, B., Lowenberg, M., and Coetzee, E. "Operational Parameter Study of Aircraft Dynamics on the Ground," Journal of Computational and Nonlinear Dynamics Vol. 5, No. 2, 2010. doi: Artn 021007

$10.1115 / 1.4000797$

20. Knowles, J. A. C., Lowenberg, M. H., Neild, S. A., and Krauskopf, B. "A bifurcation study to guide the design of a landing gear with a combined uplock/downlock mechanism," Proceedings of the Royal Society a-Mathematical Physical and Engineering Sciences Vol. 470, No. 2172, 2014.

doi: ARTN 20140332

10.1098/rspa.2014.0332

21. Whitley, D., Rana, S., and Heckendorn, R. B. "The Island Model genetic algorithm: on separability, population size and convergence," Journal of Computing and Information Technology Vol. 7, No. 1, 1999, pp. 33-47.

22. Isight Getting Started Guide. United States of America: Engineous Software, Inc., 2009, pp. 41-98. 